\title{
Entropy, Distance and Similarity Measures under Interval-Valued Intuitionistic Fuzzy Environment
}

\author{
Pratiksha Tiwari and Priti Gupta \\ Delhi Institute of Advanced Studies, Plot No. 6, Sector- 25, Rohini, Delhi, India \\ E-mail: parth12003@yahoo.co.in
}

Keywords: entropy measure, distance, similarity measure, interval valued intuitionistic fuzzy sets

Received: July 21, 2016

\begin{abstract}
This paper presents new axiomatic definitions of entropy measure using concept of probability and distance for interval-valued intuitionistic fuzzy sets (IvIFSs) by considering degree of hesitancy which is consistent with the definition of entropy given by De Luca and Termini. Thereafter, we propose some entropy measures and also derived relation between distance, entropy and similarity measures for IvIFSs. Further, we checked the performance of proposed entropy and similarity measures on the basis of intuition and compared with the existing entropy and similarity measures using numerical examples. Lastly, proposed similarity measures are used to solve problems in the field of pattern recognition and medical diagnoses.
\end{abstract}

Povzetek: V prispevku so predstavljene nove aksiomatske definicije entropijske mere za intervalno intuicionistične mehke množice.

\section{Introduction}

Fuzzy set theory (Zadeh, 1965) is tool that can handle uncertainty and imprecision effortlessly. Interval-valued, intuitionistic, interval-valued intuitionistic fuzzy sets (Zadeh (1975), Atanassov (1986), Atanassov \& Gargov (1989)), vague sets (Gau \& Buehrer, 1993) and R-fuzzy sets (Yang, Hinde, 2010) are various generalizations of Fuzzy sets (FSs). From all these generalizations IvIFSs and intuitionistic fuzzy sets (IFSs) are two conventional extensions of FSs. IvIFSs are more practical and flexible than IFSs as they are characterized by membership and non-membership degree range instead of real numbers. It makes IvIFSs more useful in dealing with real world complexities which arises due to insufficient information, lack of data, imprecise knowledge and human nature wherein range is provided instead of real numbers. Distance, entropy and similarity measures are the central arenas that are investigated by various researchers under intuitionistic and interval-valued fuzzy environment (IFE and IvFE). These measures identify the similarity or dissimilarity between two FSs. Till date, vivid entropy, distance or similarity measures are presented by various investigators. Some of these research findings are mentioned as follows: $\mathrm{Xu}$ (2007 a, b) introduced the concept of similarity between IvIFSs along with some distance measure. Zang et al. (2009) defined a entropy axiomatically for interval-valued fuzzy sets (IvFSs) and discussed relation between entropy and similarity measures. Xu and Yager (2009) studied preference relation and defined similarity measure under IvFE and interval-valued intuitionistic environment (IvIFE). Wei et al (2011) derived a generalized measure of entropy for IvIFSs. Cosine similarity measures for IvIFSs are defined by both Ye (2012) and Singh (2012). Sun \& Liu (2012), Hu \& Li (2013), Zhang et al. (2014) proposed entropy and similarity measure along with their relationship for
IvIFSs. Applications of the aforesaid entropy, distance and similarity measures are for recognition of patterns, medical diagnoses, and decision making with multiple criteria and expert systems problems. However, most of these distance, similarity or entropy measures do not consider hesitancy index between IvIFSs. Hesitance index play a very important role when membership and non-membership degree do not differ much for two IvIFSs but their hesitant index does. Some of the authors, $\mathrm{Xu}$ (2007), Xu \& Xia (2011), Wu et al. (2014) considered hesitancy into the measure of distance, similarity and entropy developed by them. Since hesitancy index also has a vital role in any decision making as it outclasses the existing methods and deals with decision process in a better way. Dammak et al. (2016) studies some possibility measures in multi-criteria decision making under IVIFE. Tiwari \& Gupta(in press) proposed generalized entropy and similarity measure for IVIFS with application in decision making. Zang et al. (2016) defined some operations on IVIFSs and proposed some aggregation operators for IvIFSs w.r.t. the restricted interval Shapley function with application in multi-criteria decision making. In this paper we have developed some of the distance, entropy and similarity measures by taking all the three degrees in account and applied it to pattern recognition and medical diagnoses under IVIFE.

This work is organized in various sections. Section 2 has basic definition and operations on IvIFSs. Section 3, presents the relationship between distance and entropy measures along with example to check the performance of entropy measures on the basis of intuition. A relation between measure of entropy and similarity measure is proposed in Section 4. Further, comparison of new similarity measures with the few existing one in done. 
Thereafter in section 5 we applied new similarity measures to recognition of patterns and medical diagnoses. Lastly conclusion is drawn in Section 6.

\section{IvIFSs along with its distance and similarity measures}

This section has definitions and concepts for IvIFSs. In this paper $\Omega=\left\{x_{1}, \ldots, x_{n}\right\}$ denotes the universe of discourse $\mathbb{C}(\Omega)$ and $\operatorname{IvIFSs}(\Omega)$ denote all crisp sets and IvIFSs respectively in $\Omega$.

Definition 1 ( Atanassov \& Gargov,1989): An IvIFS A in the finite universe $\Omega$ is defined by a triplet $\left\langle x_{i}, M V_{A}\left(x_{i}\right), N V_{A}\left(x_{i}\right), H V_{A}\left(x_{i}\right)\right\rangle \quad$ as $x_{i} \in \Omega \quad$ where $M V_{A}\left(x_{i}\right)=\left[M V_{A L}\left(x_{i}\right), M V_{A U}\left(x_{i}\right)\right]$ is called membership value interval , $N V_{A}\left(x_{i}\right)=\left[N V_{A L}\left(x_{i}\right), N V_{A U}\left(x_{i}\right)\right]$ is called non-membership value interval and $H V_{A}\left(x_{i}\right)=$ $\left[H V_{A L}\left(x_{i}\right), H V_{A U}\left(x_{i}\right)\right], H V_{A L}\left(x_{i}\right)=1-M V_{A U}\left(x_{i}\right)-$ $N V_{A U}\left(x_{i}\right), \quad H_{A U}\left(x_{i}\right)=1-M V_{A L}\left(x_{i}\right)-N V_{A L}\left(x_{i}\right)$ such that $0 \leq M V_{A U}\left(x_{i}\right)+N V_{A U}\left(x_{i}\right) \leq 1$ for each $x_{i} \in A$.

Liu (1992) defined distance and similarity measures for IvIFSs axiomatically which are given as follows:

Definition 2: For any two IvIFSs $A$ and $B$, a real valued function $\operatorname{D:} \operatorname{IvIFSs}(\Omega) \times \operatorname{IvIFSs}(\Omega) \rightarrow[0,1]$ is termed as a distance measure of IvIFSs on $\Omega$, if it satisfies the below mentioned axioms:

1. For any crisp set $A$, we have $D(A, \bar{A})=1$.

2. Distance between any two IvIFSs $A$ and $B$ is zero iff $\mathrm{A}=\mathrm{B}$.

3. Distance measure is symmetrical w.r.t to any two IvIFSs A and B.

4. For any three IvIFSs $A, B$ and $C$ such that $A \subseteq$ $\mathrm{B} \subseteq \mathrm{C}$, we $\quad$ have $\quad \mathrm{D}(\mathrm{A}, \mathrm{C}) \geq \mathrm{D}(\mathrm{A}, \mathrm{B})$ and $\mathrm{D}(\mathrm{A}, \mathrm{C}) \geq \mathrm{D}(\mathrm{B}, \mathrm{C})$.

Distance between FSs was presented by (Kacprzyk, 1997). Then its extension was proposed by Atanassov in 1999 as two dimensional distances whereas third parameter hesitancy degree in distance was introduced by Szmidt and Kacprzyk (2000) for intuitionistic fuzzy sets. Yang \& Chiclana (2012) proved three dimensional distance consistency over two dimensional distances. Grzegorzewski (2004) and Park et al. (2007) gave distance measure for IvFSs and IvIFSs respectively. Here we extend the distance measures by considering hesitancy degree for IvIFSs. For any two IvIFSs A and B, we define the following measures of distance:

1) Normalized Euclidean Distance

$$
\begin{aligned}
& \mathrm{D}_{1}(\mathrm{~A}, \mathrm{~B})=\left\{\frac { 1 } { 1 2 n } \sum _ { i = 1 } ^ { n } \left[\left(M V_{A L}\left(x_{i}\right)-\right.\right.\right. \\
& \left.M V_{B L}\left(x_{i}\right)\right)^{2}+\left(M V_{A U}\left(x_{i}\right)-M V_{B U}\left(x_{i}\right)\right)^{2}+ \\
& \left(N V_{A L}\left(x_{i}\right)-N V_{B L}\left(x_{i}\right)\right)^{2}+\left(N V_{A U}\left(x_{i}\right)-\right. \\
& \left.N V_{B U}\left(x_{i}\right)\right)^{2}+\left(H V_{A L}\left(x_{i}\right)-H V_{B L}\left(x_{i}\right)\right)^{2}+ \\
& \left.\left.\left(H V_{A U}\left(x_{i}\right)-H V_{B U}\left(x_{i}\right)\right)^{2}\right]\right\}^{1 / 2}
\end{aligned}
$$

2) Normalized Hamming Distance

$$
\begin{aligned}
& \mathrm{D}_{2}(\mathrm{~A}, \mathrm{~B})=\frac{1}{8 \mathrm{n}} \sum_{i=1}^{n}\left[\left|M V_{A L}\left(x_{i}\right)-M V_{B L}\left(x_{i}\right)\right|+\right. \\
& \left|M V_{A U}\left(x_{i}\right)-M V_{B U}\left(x_{i}\right)\right|+\mid N V_{A L}\left(x_{i}\right)- \\
& N V_{B L}\left(x_{i}\right)|+| N V_{A U}\left(x_{i}\right)-N V_{B U}\left(x_{i}\right) \mid+ \\
& \left|H V_{A L}\left(x_{i}\right)-H V_{B L}\left(x_{i}\right)\right|+\mid H V_{A U}\left(x_{i}\right)- \\
& \left.H V_{B U}\left(x_{i}\right) \mid\right]
\end{aligned}
$$

3) Hamming Hausdorff Normalized Distance

$$
\begin{aligned}
& \mathrm{D}_{3}(\mathrm{~A}, \mathrm{~B})=\frac{1}{4 \mathrm{n}} \sum_{i=1}^{n}\left[\left|M V_{A L}\left(x_{i}\right)-M V_{B L}\left(x_{i}\right)\right| \mathrm{\vee}\right. \\
& \left|M V_{A U}\left(x_{i}\right)-M V_{B U}\left(x_{i}\right)\right|+\mid N V_{A L}\left(x_{i}\right)- \\
& N V_{B L}\left(x_{i}\right)|\vee| N V_{A U}\left(x_{i}\right)-N V_{B U}\left(x_{i}\right) \mid+ \\
& \left|H V_{A L}\left(x_{i}\right)-H V_{B L}\left(x_{i}\right)\right| \vee \mid H V_{A U}\left(x_{i}\right)- \\
& \left.H V_{B U}\left(x_{i}\right) \mid\right]
\end{aligned}
$$

4) Hausdorff Normalized Hamming Distance $\mathrm{D}_{4}(\mathrm{~A}, \mathrm{~B})=$

$\frac{1}{4 \mathrm{n}} \sum_{i=1}^{n} \max \left\{\begin{array}{l}\frac{\left|M V_{A L}\left(x_{i}\right)-M V_{B L}\left(x_{i}\right)\right|+\left|M V_{A U}\left(x_{i}\right)-M V_{B U}\left(x_{i}\right)\right|}{2}, \\ \frac{\left|N V_{A L}\left(x_{i}\right)-N V_{B L}\left(x_{i}\right)\right|+\left|N V_{A U}\left(x_{i}\right)-N V_{B U}\left(x_{i}\right)\right|}{2}, \\ \frac{\left|H V_{A L}\left(x_{i}\right)-H V_{B L}\left(x_{i}\right)\right|+\left|H V_{A U}\left(x_{i}\right)-H V_{B U}\left(x_{i}\right)\right|}{2}\end{array}\right\}$

5) Averaged fifth Distance Measure

$\mathrm{D}_{5}(\mathrm{~A}, \mathrm{~B})=$

$\frac{1}{2 \mathrm{n}} \sum_{i=1}^{n}\left\{\begin{array}{l}{\left[\begin{array}{l}\left|M V_{A L}\left(x_{i}\right)-M V_{B L}\left(x_{i}\right)\right|+\left|M V_{A U}\left(x_{i}\right)-M V_{B U}\left(x_{i}\right)\right| \\ +\left|N V_{A L}\left(x_{i}\right)-N V_{B L}\left(x_{i}\right)\right|+\left|N V_{A U}\left(x_{i}\right)-N V_{B U}\left(x_{i}\right)\right| \\ +\left|H V_{A L}\left(x_{i}\right)-H V_{B L}\left(x_{i}\right)\right|+\left|H V_{A U}\left(x_{i}\right)-H V_{B U}\left(x_{i}\right)\right|\end{array}\right]} \\ 8\end{array}\right.$
$\left.\frac{\max \left(\begin{array}{c}\left|M V_{A L}\left(x_{i}\right)-M V_{B L}\left(x_{i}\right)\right|+\left|M V_{A U}\left(x_{i}\right)-M V_{B U}\left(x_{i}\right)\right|, \\ \left|N V_{A L}\left(x_{i}\right)-N V_{B L}\left(x_{i}\right)\right|+\left|N V_{A U}\left(x_{i}\right)-N V_{B U}\left(x_{i}\right)\right| \\ \left|H V_{A L}\left(x_{i}\right)-H V_{B L}\left(x_{i}\right)\right|+\left|H V_{A U}\left(x_{i}\right)-H V_{B U}\left(x_{i}\right)\right|\end{array}\right)}{4}\right\}$

6) Generalized Measure of Distance, for $\mathrm{p} \geq 2$,

$$
\begin{aligned}
& \mathrm{D}_{6}(\mathrm{~A}, \mathrm{~B})=\left\{\frac { 1 } { 1 2 \mathrm { n } } \sum _ { i = 1 } ^ { n } \left(\left|M V_{A L}\left(x_{i}\right)-M V_{B L}\left(x_{i}\right)\right| \mathrm{\vee}\right.\right. \\
& \left.\left|M V_{A U}\left(x_{i}\right)-M V_{B U}\left(x_{i}\right)\right|\right)^{p}+\left(\mid N V_{A L}\left(x_{i}\right)-\right. \\
& \left.N V_{B L}\left(x_{i}\right)|\vee| N V_{A U}\left(x_{i}\right)-N V_{B U}\left(x_{i}\right) \mid\right)^{p}+ \\
& \left(\left|H V_{A L}\left(x_{i}\right)-H V_{B L}\left(x_{i}\right)\right| \vee \mid H V_{A U}\left(x_{i}\right)-\right. \\
& \left.\left.H V_{B U}\left(x_{i}\right) \mid\right)^{p}\right\}^{1 / \mathrm{p}}
\end{aligned}
$$

Definition 3: Let A and B be any two IvIFSs, a real valued function $\mathrm{S}: \operatorname{IvIFSs}(\Omega) \times \operatorname{IvIFSs}(\Omega) \rightarrow[0,1]$ is defined as a measure of similarity for IvIFSs on $\Omega$, if it satisfies axioms mentioned below:

1. For any crisp set $A$, we have $S(A, \bar{A})=0$

2. Measure of similarity between any two IvIFSs is 1 iff $A=B$.

3. Measure of similarity is symmetric w.r.t. any two IvIFSs.

4. For any three IvIFSs $A, B$ and $C$ such that $A \subseteq$ $\mathrm{B} \subseteq \mathrm{C} . \quad$ We have $\mathrm{S}(\mathrm{A}, \mathrm{C}) \leq \mathrm{S}(\mathrm{A}, \mathrm{B})$ and $\mathrm{S}(\mathrm{A}, \mathrm{C}) \leq \mathrm{S}(\mathrm{B}, \mathrm{C})$. 
From axiomatic definition of distance and similarity measures it is clear that $\mathrm{S}(\mathrm{A}, \mathrm{B})=1-\mathrm{D}(\mathrm{A}, \mathrm{B})$ where $\mathrm{A}$ and $B$ are IvIFSs, $D$ and $S$ are distance and similarity measure for IvIFSs respectively.

\subsection{Entropy measure for IvIFSs}

In 1972, De Luca and Termini defined measure of entropy for FSs. Hung \& Yang (2006) extended definition for IFSs considering hesitancy degree. The following definition for entropy is an extension of definition of entropy proposed by Hung \& Yang (2006) for IvIFSs.

Definition 4: A real valued function $\mathrm{E}: \operatorname{IvIFSs}(\Omega) \rightarrow$ $[0,1]$ is termed as measure of entropy under IvIFE, if below mentioned axioms are satisfied:

1. $\mathrm{E}(\mathrm{A})=0, \forall \mathrm{A} \in \mathbb{C}(\Omega)$;

2. $\mathrm{E}(\mathrm{A})=1$, iff $M_{A}\left(x_{i}\right)=N_{A}\left(x_{i}\right)=H_{A}\left(x_{i}\right)=$ $\left[\frac{1}{3}, \frac{1}{3}\right], \forall x_{i} \in \Omega$;

3. $E(A) \leq E(B)$, if $A$ is less fuzzy than $B$;

4. $\mathrm{E}(\mathrm{A})=\mathrm{E}(\overline{\mathrm{A}})$, where $\overline{\mathrm{A}}$ is complement of $\mathrm{A}$, where $A, B \in \operatorname{IvIFSs}(\Omega)$.

Above definition is steady with description of measure of entropy given by De Luca \& Termini (1972). As it is known that complete description of an IvIFS $A \in \Omega$ has three degrees membership, non-membership and hesitancy with $M V_{A U}\left(x_{i}\right)+N V_{A U}\left(x_{i}\right)+H V_{A L}\left(x_{i}\right)=1$ and $\quad M V_{A L}\left(x_{i}\right)+N V_{A L}\left(x_{i}\right)+H V_{A U}\left(x_{i}\right)=1 \quad$ with $0 \leq M V_{A U}\left(x_{i}\right), N V_{A U}\left(x_{i}\right), H V_{A L}\left(x_{i}\right)$,

$M V_{A L}\left(x_{i}\right), N V_{A L}\left(x_{i}\right), H V_{A U}\left(x_{i}\right) \leq 1$. By taking all the three in to consideration we may assume them as probability measure. Therefore the entropy is maximum when all the variables are equal (i.e. $M V_{A U}\left(x_{i}\right)=$ $N V_{A U}\left(x_{i}\right)=H V_{A L}\left(x_{i}\right)=\frac{1}{3}$ and $M V_{A L}\left(x_{i}\right)=N V_{A L}\left(x_{i}\right)=$ $H V_{A U}\left(x_{i}\right)=\frac{1}{3}$ ) and zero (minimum) when only one variable is exists (i.e. $M V_{A L}\left(x_{i}\right)=M V_{A U}\left(x_{i}\right)=$ 1, $N V_{A L}\left(x_{i}\right)=N V_{A U}\left(x_{i}\right)=0, H V_{A L}\left(x_{i}\right)=H V_{A U}\left(x_{i}\right)=0$ or $M V_{A L}\left(x_{i}\right)=M V_{A U}\left(x_{i}\right)=0, N V_{A L}\left(x_{i}\right)=N V_{A U}\left(x_{i}\right)=$ $1, H V_{A L}\left(x_{i}\right)=H V_{A U}\left(x_{i}\right)=1$

or $\quad M V_{A L}\left(x_{i}\right)=M V_{A U}\left(x_{i}\right)=0, N V_{A L}\left(x_{i}\right)=$ $\left.N V_{A U}\left(x_{i}\right)=0, H V_{A L}\left(x_{i}\right)=H V_{A U}\left(x_{i}\right)=1\right)$.

Again we extend the definition of entropy given by Zang et al. (2014) based on distance for IvIFSs. In following definition we have considered degree of hesitation, which is not considered by other definitions of entropy.

Definition 5: A real-valued function $\operatorname{E}: \operatorname{IvIFSs}(\Omega) \rightarrow$ $[0,1]$ is termed as measure of entropy under IvIFE, if the following axioms are satisfied:

1. $\mathrm{E}(\mathrm{A})=0, \forall \mathrm{A} \in \mathbb{C}(\Omega)$;

2. $\mathrm{E}(\mathrm{A})=1$, iff all the three description of IvIFSs intervals satifies $\quad M V_{A}\left(x_{i}\right)=N V_{A}\left(x_{i}\right)=$ $H V_{A}\left(x_{i}\right)=\left[\frac{1}{3}, \frac{1}{3}\right], \forall x_{i} \in \Omega$

3. If $\mathrm{D}\left(\mathrm{A},\left\langle\left[\frac{1}{3}, \frac{1}{3}\right],\left[\frac{1}{3}, \frac{1}{3}\right],\left[\frac{1}{3}, \frac{1}{3}\right]\right\rangle\right) \geq$ $\mathrm{D}\left(\mathrm{B},\left\langle\left[\frac{1}{3}, \frac{1}{3}\right],\left[\frac{1}{3}, \frac{1}{3}\right],\left[\frac{1}{3}, \frac{1}{3}\right]\right\rangle\right)$, then $\mathrm{E}(\mathrm{A}) \leq \mathrm{E}(\mathrm{B})$, $\forall \mathrm{A}, \mathrm{B} \in \operatorname{IvIFSs}(\Omega)$, where $\mathrm{D}$ is measure of distance.

4. $\mathrm{E}(\mathrm{A})=\mathrm{E}(\overline{\mathrm{A}})$, where $\overline{\mathrm{A}}$ is complement of $\mathrm{A}$, where $A, B \in \operatorname{IvIFSs}(\Omega)$.

In the next section, we derive a relation which relates measure of distance and entropy for IvIFSs, which satisfies all the axioms of the definition of entropy.

\section{Relation between measure of distance and entropy}

Here, we develop a technique which obtains entropy measure for IvIFSs which satisfies the aforementioned properties.

Theorem 2: Let $D_{j}, j=1, \ldots, 6$ be the above-mentioned six distance measure equations (1)-(6) between IvIFSs, then, $\quad E_{j}(A)=1-3 D_{j}\left(A,\left\langle\left[\frac{1}{3}, \frac{1}{3}\right],\left[\frac{1}{3}, \frac{1}{3}\right],\left[\frac{1}{3}, \frac{1}{3}\right]\right\rangle\right), \quad j=$ $1, \ldots, 6$ for any $A \in \operatorname{IvIFSs}(\Omega)$ are measure of entropy of IvIFSs.

Proof: We prove that $\mathrm{E}_{j}(\mathrm{~A})$, for $\mathrm{j}=1, \ldots, 6$ satisfies conditions given by definition 5 .

Property1): If $\mathrm{A} \in \mathbb{C}(\Omega) \Rightarrow A\left(x_{i}\right)=\langle[1,1],[0,0],[0,0]\rangle$ or $A\left(x_{i}\right)=\langle[0,0],[1,1],[0,0]\rangle, \forall x_{i} \in \Omega$, then for $\mathrm{j}=$ $1, \ldots, 6$

Thus, $E_{j}(A)=0$

$$
D_{j}\left(A,\left\langle\left[\frac{1}{3}, \frac{1}{3}\right],\left[\frac{1}{3}, \frac{1}{3}\right],\left[\frac{1}{3}, \frac{1}{3}\right]\right\rangle\right)=\frac{1}{3} .
$$

Property 2): For all $\mathrm{j}=1, \ldots, 6, \mathrm{E}_{\mathrm{j}}(\mathrm{A})=1$

$\Leftrightarrow 1-3 \mathrm{D}_{\mathrm{j}}\left(\mathrm{A},\left\langle\left[\frac{1}{3}, \frac{1}{3}\right],\left[\frac{1}{3}, \frac{1}{3}\right],\left[\frac{1}{3}, \frac{1}{3}\right]\right\rangle\right)=1$

$\Leftrightarrow 3 \mathrm{D}_{j}\left(\mathrm{~A},\left\langle\left[\frac{1}{3}, \frac{1}{3}\right],\left[\frac{1}{3}, \frac{1}{3}\right],\left[\frac{1}{3}, \frac{1}{3}\right]\right\rangle\right)=0$

$\Leftrightarrow \mathrm{A}=\left\langle\left[\frac{1}{3}, \frac{1}{3}\right],\left[\frac{1}{3}, \frac{1}{3}\right],\left[\frac{1}{3}, \frac{1}{3}\right]\right\rangle$

Property3): Let A and B be any two IvIFSs and $\mathrm{D}_{\mathrm{j}}\left(\mathrm{A},\left\langle\left[\frac{1}{3}, \frac{1}{3}\right],\left[\frac{1}{3}, \frac{1}{3}\right],\left[\frac{1}{3}, \frac{1}{3}\right]\right\rangle\right) \geq$

$D_{j}\left(B,\left\langle\left[\frac{1}{3}, \frac{1}{3}\right],\left[\frac{1}{3}, \frac{1}{3}\right],\left[\frac{1}{3}, \frac{1}{3}\right]\right\rangle\right)$ then

$$
\begin{aligned}
1-3 D_{j}\left(A,\left\langle\left[\frac{1}{3}, \frac{1}{3}\right],\left[\frac{1}{3}, \frac{1}{3}\right],\left[\frac{1}{3}, \frac{1}{3}\right]\right\rangle\right) \\
\geq 1-3 D_{j}\left(B,\left\langle\left[\frac{1}{3}, \frac{1}{3}\right],\left[\frac{1}{3}, \frac{1}{3}\right],\left[\frac{1}{3}, \frac{1}{3}\right]\right\rangle\right)
\end{aligned}
$$

$\Rightarrow E_{j}(A) \leq E_{j}(B)$, for all $j=1, \ldots, 6$

Property 4) : Let $\mathrm{A}$ be any IVIFS then $\overline{\mathrm{A}}=$ $\left\{\left\langle x_{i},\left[N V_{A L}\left(x_{i}\right), N V_{A U}\left(x_{i}\right)\right],\left[M V_{A L}\left(x_{i}\right), M V_{A U}\left(x_{i}\right)\right]\right\rangle / x_{i} \in \Omega\right\}$ $\Rightarrow \mathrm{D}_{\mathrm{j}}\left(\mathrm{A},\left\langle\left[\frac{1}{3}, \frac{1}{3}\right],\left[\frac{1}{3}, \frac{1}{3}\right],\left[\frac{1}{3}, \frac{1}{3}\right]\right\rangle\right)$ $=\mathrm{D}_{j}\left(\overline{\mathrm{A}},\left\langle\left[\frac{1}{3}, \frac{1}{3}\right],\left[\frac{1}{3}, \frac{1}{3}\right],\left[\frac{1}{3}, \frac{1}{3}\right]\right\rangle\right)$

Thus, $E_{j}(A)=E_{j}(\bar{A})$, for all $j=1, \ldots, 6$.

From theorem 2 and various distance formulas' mentioned (equation (1) to (6)), we get corresponding entropy formulas as follows: 


$$
\begin{aligned}
& E_{1}(A)=1-3\left\{\frac { 1 } { 1 2 n } \sum _ { i = 1 } ^ { n } \left[\left(M V_{A L}\left(x_{i}\right)-\frac{1}{3}\right)^{2}+\right.\right. \\
& \left(M V_{A U}\left(x_{i}\right)-\frac{1}{3}\right)^{2}+\left(N V_{A L}\left(x_{i}\right)-\frac{1}{3}\right)^{2}+ \\
& \left(N V_{A U}\left(x_{i}\right)-\frac{1}{3}\right)^{2}+\left(H V_{A L}\left(x_{i}\right)-\frac{1}{3}\right)^{2}+ \\
& \left.\left.\left(H V_{A U}\left(x_{i}\right)-\frac{1}{3}\right)^{2}\right]\right\}^{1 / 2} \\
& E_{2}(A)=1-\frac{3}{8 n} \sum_{i=1}^{n}\left[\left|M V_{A L}\left(x_{i}\right)-\frac{1}{3}\right|+\right. \\
& \left|M V_{A U}\left(x_{i}\right)-\frac{1}{3}\right|+\left|N V_{A L}\left(x_{i}\right)-\frac{1}{3}\right|+ \\
& \left|N V_{A U}\left(x_{i}\right)-\frac{1}{3}\right|+\left|H V_{A L}\left(x_{i}\right)-\frac{1}{3}\right|+ \\
& \left.\left|H V_{A U}\left(x_{i}\right)-\frac{1}{3}\right|\right] \\
& E_{3}(A)=1-\frac{3}{4 n} \sum_{i=1}^{n}\left[\left|M V_{A L}\left(x_{i}\right)-\frac{1}{3}\right| \mathrm{V}\right. \\
& \left|M V_{A U}\left(x_{i}\right)-\frac{1}{3}\right|+\left|N V_{A L}\left(x_{i}\right)-\frac{1}{3}\right| \mathrm{v} \\
& \left|N V_{A U}\left(x_{i}\right)-\frac{1}{3}\right|+\left|H V_{A L}\left(x_{i}\right)-\frac{1}{3}\right| \mathrm{v} \\
& \left.\left|H V_{A U}\left(x_{i}\right)-\frac{1}{3}\right|\right] \\
& E_{4}(A)=1- \\
& \frac{3}{2 n} \sum_{i=1}^{n} \max \left\{\begin{array}{l}
\frac{\left|M V_{A L}\left(x_{i}\right)-1 / 3\right|+\left|M V_{A U}\left(x_{i}\right)-1 / 3\right|}{2}, \\
\frac{\left|N V_{A L}\left(x_{i}\right)-1 / 3\right|+\left|N V_{A U}\left(x_{i}\right)-1 / 3\right|}{2}, \\
\frac{\left|H V_{A L}\left(x_{i}\right)-1 / 3\right|+\left|H V_{A U}\left(x_{i}\right)-1 / 3\right|}{2}
\end{array}\right\}
\end{aligned}
$$$$
=1
$$$$
\begin{aligned}
& -\frac{3}{2 \mathrm{n}} \sum_{\mathrm{i}=1}^{\mathrm{n}}\left\{\begin{array}{l}
{\left[\begin{array}{l}
\left|M V_{A L}\left(x_{i}\right)-1 / 3\right|+\left|M V_{A U}\left(x_{i}\right)-1 / 3\right| \\
+\left|N V_{A L}\left(x_{i}\right)-1 / 3\right|+\left|N V_{A U}\left(x_{i}\right)-1 / 3\right| \\
+\left|H V_{A L}\left(x_{i}\right)-1 / 3\right|+\left|H V_{A U}\left(x_{i}\right)-1 / 3\right|
\end{array}\right]} \\
8
\end{array}\right. \\
& \left.+\frac{\max \left(\begin{array}{l}
\left|M V_{A L}\left(x_{i}\right)-1 / 3\right|+\left|M V_{A U}\left(x_{i}\right)-1 / 3\right|, \\
\left|N V_{A L}\left(x_{i}\right)-1 / 3\right|+\left|N V_{A U}\left(x_{i}\right)-1 / 3\right|, \\
\left|H V_{A L}\left(x_{i}\right)-1 / 3\right|+\left|H V_{A U}\left(x_{i}\right)-1 / 3\right|
\end{array}\right)}{2}\right\}
\end{aligned}
$$

$$
\begin{aligned}
& E_{6}(A, B)=1-3 \sum_{i=1}^{n}\left[\frac { 1 } { 1 2 n } \left[\left(\left|M V_{A L}\left(x_{i}\right)-\frac{1}{3}\right| \vee\right.\right.\right. \\
& \left.\left|M V_{A U}\left(x_{i}\right)-\frac{1}{3}\right|\right)^{p}+\left(\left|N V_{A L}\left(x_{i}\right)-\frac{1}{3}\right| \vee\right. \\
& \left.\left|N V_{A U}\left(x_{i}\right)-\frac{1}{3}\right|\right)^{p}+\left(\left|H V_{A L}\left(x_{i}\right)-\frac{1}{3}\right| \vee\right. \\
& \left.\left.\left.\left|H V_{A U}\left(x_{i}\right)-\frac{1}{3}\right|\right)^{p}\right]\right]^{1 / p}
\end{aligned}
$$

To check the consistency of proposed entropy measures with the intuitionist beliefs we have used the following example.
Example: Consider two IvIFSs $A=$ $\{x,\langle[0.2,0.2],[0.2,0.3],[0.5,0.6]\rangle, x \in \Omega\}$ and $\quad B=$ $\{x,\langle[0.2,0.3],[0.4,0.6],[0.1,0.4]\rangle, x \in \Omega\}$, clearly we can see that $\mathrm{A}$ is more fuzzy than $\mathrm{B}$. Then $\mathrm{E}_{j}(\mathrm{~A})$ and $\mathrm{E}_{j}(\mathrm{~B})$ are given in Table 1:

\begin{tabular}{|c|l|l|}
\hline Entropies & \multicolumn{1}{|c|}{ A } & \multicolumn{1}{c|}{ B } \\
\hline $\mathrm{E}_{1}$ & 0.5570 & 0.65866 \\
\hline $\mathrm{E}_{2}$ & 0.675 & 0.7 \\
\hline $\mathrm{E}_{3}$ & 0.6 & 0.525 \\
\hline $\mathrm{E}_{4}$ & 0.675 & 0.75 \\
\hline $\mathrm{E}_{5}$ & 0.5125 & 0.6 \\
\hline $\mathrm{E}_{6}$ & 0.7171 & 0.672 \\
\hline
\end{tabular}

Table 1: Entropies.

Since $E_{3}(A)>E_{3}(B)$ and $E_{6}(A)>E_{6}(B)$ which indicates that $E_{3}$ and $E_{6}$ are consistent with the intuition.

\subsection{Comparison of existing entropy measure with proposed entropy measures}

We compared performance of existing entropy measures with the proposed measures with the help of an example. Let $\mathrm{A}$ be an IvIFS, then

$$
E_{Z J}(A)=
$$

$\frac{1}{n} \sum_{=1}^{n} \frac{\min \left(M V_{A L}\left(x_{i}\right), N V_{A L}\left(x_{i}\right)\right)+\min \left(M V_{A U}\left(x_{i}\right), N V_{A U}\left(x_{i}\right)\right)}{\max \left(M V_{A L}\left(x_{i}\right), N V_{A L}\left(x_{i}\right)\right)+\max \left(M V_{A U}\left(x_{i}\right), N V_{A U}\left(x_{i}\right)\right)}$

$$
\begin{aligned}
& E_{W W}(A)=
\end{aligned}
$$

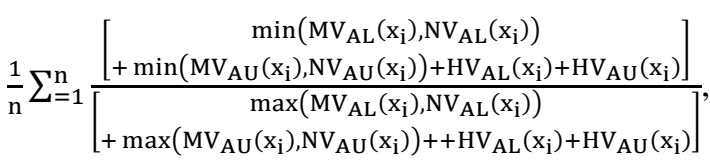

$$
\begin{aligned}
& E_{Z M}(A)=\frac{1}{n} \sum_{i=1}^{n}\left[1-\left(\overline{M V}_{A}\left(x_{i}\right)+\right.\right. \\
& \left.\left.\overline{N V}_{A}\left(x_{i}\right)\right) e^{1-\left(\overline{M V}_{A}\left(x_{i}\right)+\overline{N V}_{A}\left(x_{i}\right)\right)}\right] \text {, }
\end{aligned}
$$

where $\overline{M V}_{A}\left(x_{i}\right)=M V_{A L}\left(x_{i}\right)+\tau \Delta M V_{A}\left(x_{i}\right), \quad \overline{N V}_{A}\left(x_{i}\right)=$ $N V_{A L}\left(x_{i}\right)+\tau \Delta N V_{A}\left(x_{i}\right)$ and $\Delta M V_{A}\left(x_{i}\right)=M V_{A U}\left(x_{i}\right)-$ $M V_{A L}\left(x_{i}\right)$ and $\Delta N V_{A}\left(x_{i}\right)=N V_{A U}\left(x_{i}\right)-N V_{A L}\left(x_{i}\right), \tau \in$ $[0,1]$

are the measures of entropies given by Zang et al. (2010), Wei et al.(2011) and Zang et al. (2011) respectively.

Example: Consider the example from Sun \& Liu (2012) to review the entropies for an IvIFSs $A_{i}$ which are as follows:

$$
\begin{aligned}
& “ A_{1}=\{x,\langle[0.1,0.2],[0.2,0.4],[0.4,0.7]\rangle, x \in \Omega\}, \\
& A_{2}=\{x,\langle[0.2,0.2],[0.3,0.5],[0.3,0.5]\rangle, x \in \Omega\}, \\
& A_{3}=\{x,\langle[0.2,0.4],[0,0],[0.6,0.8]\rangle, x \in \Omega\}, \\
& A_{4}=\{x,\langle[0.3,0.4],[0,0.142857],[0.4571,0.7]\rangle, x \in \Omega\}, \\
& A_{5}=\{x,\langle[0.1,0.1],[0,0.2],[0.6,0.9]\rangle, x \in \Omega\}, \\
& A_{6}=\{x,\langle[0,0.2],[0,0.2],[0.4,1.0]\rangle, x \in \Omega\},
\end{aligned}
$$




\begin{tabular}{|l|l|l|l|l|l|l|}
\hline & $E_{1}\left(A_{i}\right)$ & $E_{2}\left(A_{i}\right)$ & $E_{3}\left(A_{i}\right)$ & $E_{4}\left(A_{i}\right)$ & $E_{5}\left(A_{i}\right)$ & $E_{6}\left(A_{i}\right)$ \\
\hline $\mathrm{A}_{1}$ & 0.58167 & 0.625 & 0.45 & 0.675 & 0.4875 & 0.6063 \\
\hline $\mathrm{A}_{2}$ & 0.735425 & 0.75 & 0.65 & 0.8 & 0.675 & 0.765479 \\
\hline $\mathrm{A}_{3}$ & 0.367544 & 0.4 & 0.3 & 0.45 & 0.15 & 0.490098 \\
\hline $\mathrm{A}_{4}$ & 0.523512 & 0.582143 & 0.425 & 0.607143 & 0.398214 & 0.566987 \\
\hline $\mathrm{A}_{5}$ & 0.27889 & 0.3 & 0.15 & 0.3 & 0.05 & 0.395848 \\
\hline $\mathrm{A}_{6}$ & 0.271989 & 0.375 & 0.01 & 0.45 & 0.1375 & 0.292893 \\
\hline
\end{tabular}

Table 2: Comparison of entropies.

The values of $E_{Z J}\left(A_{1}\right)=0.5=E_{Z J}\left(A_{2}\right)$, $E_{W W}\left(A_{3}\right)=0.7=E_{W W}\left(A_{4}\right)$ and $E_{Z M}\left(A_{5}\right)=0.5=$ $E_{Z M}\left(A_{6}\right)$. Thus, the entropies $E_{Z J}\left(A_{i}\right), E_{W W}\left(A_{i}\right)$ and $E_{Z M}\left(A_{i}\right)$ are unreasonable. Proposed entropies $E_{\mathrm{j}}, \mathrm{j}=$ $1,2, . .6$ can discriminate the fuzziness of all the IvIFSs $A_{i}, i=1, \ldots, 6$ given as follows and give the reasonable results given in Table 2 .

Here we have proposed some entropy measures and evaluated its performance on the basis of intuitionistic belief and comparison with existing measures. In next section we propose relation between measures of entropy and similarity under IvIFE. Also, we have defined new measures of similarity and have evaluated their performance by comparing them with some existing measures.

\section{Relations between measure of entropy and similarity together with new similarity measures}

In this section contains definition of new measures of similarity under IvIFE and determined an important relation between entropy and similarity measure IvIFSs which is discussed as follows.

Theorem 3: Let $S_{j}$, for $j=1, \ldots, 6$ be measure of similarity of IvIFSs w.r.t. the measure of distance $D_{j}$, for $j=1, \ldots, 6$ respectively, and $\mathrm{A}$ be any IvIFS. Then $E_{j}(A)=3 S_{j}\left(A,\left\langle\left[\frac{1}{3}, \frac{1}{3}\right],\left[\frac{1}{3}, \frac{1}{3}\right],\left[\frac{1}{3}, \frac{1}{3}\right]\right\rangle\right)-2, \quad$ for $\quad j=$ $1, \ldots, 6$ are measures of entropy for IvIFSs.

Proof: We prove that $E_{j}(A)$, for $j=1, \ldots, 6$ satisfies conditions given by definition 5 .

Property 1): If $\mathrm{A} \in \mathbb{C}(\Omega) \Rightarrow A\left(x_{i}\right)=\langle[1,1],[0,0],[0,0]\rangle$ or $A\left(x_{i}\right)=\langle[0,0],[1,1],[0,0]\rangle, \forall x_{i} \in \Omega$, then for $j=$ $1, \ldots, 6$

$$
\begin{aligned}
S_{j}\left(A,\left\langle\left[\frac{1}{3}, \frac{1}{3}\right],\left[\frac{1}{3},\right.\right.\right. & \left.\left.\left.\frac{1}{3}\right],\left[\frac{1}{3}, \frac{1}{3}\right]\right\rangle\right) \\
& =1-D_{j}\left(A,\left\langle\left[\frac{1}{3}, \frac{1}{3}\right],\left[\frac{1}{3}, \frac{1}{3}\right],\left[\frac{1}{3}, \frac{1}{3}\right]\right\rangle\right) \\
& =\frac{2}{3} .
\end{aligned}
$$

Thus, $E_{j}(A)=0$

Property 2): For all $j=1, \ldots, 6, E_{j}(A)=1$

$\Leftrightarrow 3 \mathrm{~S}_{\mathrm{j}}\left(\mathrm{A},\left\langle\left[\frac{1}{3}, \frac{1}{3}\right],\left[\frac{1}{3}, \frac{1}{3}\right],\left[\frac{1}{3}, \frac{1}{3}\right]\right\rangle\right)-2=1$

$$
\begin{aligned}
& \Leftrightarrow S_{j}\left(A,\left\langle\left[\frac{1}{3}, \frac{1}{3}\right],\left[\frac{1}{3}, \frac{1}{3}\right],\left[\frac{1}{3}, \frac{1}{3}\right]\right\rangle\right)=1 \\
& \Leftrightarrow A=\left\langle\left[\frac{1}{3}, \frac{1}{3}\right],\left[\frac{1}{3}, \frac{1}{3}\right],\left[\frac{1}{3}, \frac{1}{3}\right]\right\rangle
\end{aligned}
$$

Property3): Let $A$ and $B$ be any two IvIFSs and $\mathrm{D}_{\mathrm{j}}\left(\mathrm{A},\left\langle\left[\frac{1}{3}, \frac{1}{3}\right],\left[\frac{1}{3}, \frac{1}{3}\right],\left[\frac{1}{3}, \frac{1}{3}\right]\right\rangle\right) \geq$ $D_{j}\left(B,\left\langle\left[\frac{1}{3}, \frac{1}{3}\right],\left[\frac{1}{3}, \frac{1}{3}\right],\left[\frac{1}{3}, \frac{1}{3}\right]\right\rangle\right)$ then

$$
\begin{aligned}
1-\mathrm{D}_{j}\left(\mathrm{~A},\left\langle\left[\frac{1}{3}, \frac{1}{3}\right]\right.\right. & \left.\left.,\left[\frac{1}{3}, \frac{1}{3}\right],\left[\frac{1}{3}, \frac{1}{3}\right]\right\rangle\right) \\
& \leq 1-\mathrm{D}_{j}\left(\mathrm{~B},\left\langle\left[\frac{1}{3}, \frac{1}{3}\right],\left[\frac{1}{3}, \frac{1}{3}\right],\left[\frac{1}{3}, \frac{1}{3}\right]\right\rangle\right) \\
\Leftrightarrow \mathrm{S}_{\mathrm{j}}\left(\mathrm{A},\left\langle\left[\frac{1}{3}, \frac{1}{3}\right]\right.\right. & \left.\left.,\left[\frac{1}{3}, \frac{1}{3}\right],\left[\frac{1}{3}, \frac{1}{3}\right]\right\rangle\right) \\
& \leq \mathrm{S}_{j}\left(\mathrm{~B},\left\langle\left[\frac{1}{3}, \frac{1}{3}\right],\left[\frac{1}{3}, \frac{1}{3}\right],\left[\frac{1}{3}, \frac{1}{3}\right]\right\rangle\right)
\end{aligned}
$$$$
\Leftrightarrow \mathrm{E}_{j}(\mathrm{~A}) \leq \mathrm{E}_{\mathrm{j}}(\mathrm{B}) \text {, for all } j=1, \ldots, 6
$$

Property 4) : Let $\mathrm{A}$ be any IvIFS then $\overline{\mathrm{A}}=$ $\left\{\left\langle x_{i},\left[N V_{A L}\left(x_{i}\right), N V_{A U}\left(x_{i}\right)\right],\left[M V_{A L}\left(x_{i}\right), M V_{A U}\left(x_{i}\right)\right]\right\rangle / x_{i} \in \Omega\right\}$ $\Rightarrow D_{j}\left(\mathrm{~A},\left\langle\left[\frac{1}{3}, \frac{1}{3}\right],\left[\frac{1}{3}, \frac{1}{3}\right],\left[\frac{1}{3}, \frac{1}{3}\right]\right\rangle\right)$

$$
\begin{aligned}
&=\mathrm{D}_{\mathrm{j}}\left(\overline{\mathrm{A}},\left\langle\left[\frac{1}{3}, \frac{1}{3}\right],\left[\frac{1}{3}, \frac{1}{3}\right],\left[\frac{1}{3}, \frac{1}{3}\right]\right\rangle\right) \\
& \Rightarrow \mathrm{S}_{j}\left(\mathrm{~A},\left\langle\left[\frac{1}{3}, \frac{1}{3}\right],\right.\right.\left.\left.,\left[\frac{1}{3}, \frac{1}{3}\right],\left[\frac{1}{3}, \frac{1}{3}\right]\right\rangle\right) \\
&=\mathrm{S}_{j}\left(\overline{\mathrm{A}},\left\langle\left[\frac{1}{3}, \frac{1}{3}\right],\left[\frac{1}{3}, \frac{1}{3}\right],\left[\frac{1}{3}, \frac{1}{3}\right]\right\rangle\right)
\end{aligned}
$$

Thus, $E_{j}(A)=E_{j}(\bar{A})$, for all $j=1, \ldots, 6$.

Next, we present a conversion technique to define similarity measures established by entropy measure for IvIFSs.

Definition 6 : For any two IvIFSs $\mathrm{A}$ and $\mathrm{B}$ in $\Omega$, such that both $\mathrm{A}$ and $\mathrm{B}$ are defined by the triplet $\left\langle x_{i},\left[M V_{A L}\left(x_{i}\right), M V_{A U}\left(x_{i}\right)\right],\left[N V_{A L}\left(x_{i}\right), N V_{A U}\left(x_{i}\right)\right]\right\rangle \quad$ and $\left\langle x_{i},\left[M V_{B L}\left(x_{i}\right), M V_{B U}\left(x_{i}\right)\right],\left[N V_{B L}\left(x_{i}\right), N V_{B U}\left(x_{i}\right)\right]\right\rangle$ respec tively. we define an IvIFSs $\varnothing(\mathrm{A}, \mathrm{B})$ using $\mathrm{A}$ and B as given below:

$$
\begin{aligned}
& M V_{\varnothing(A, B) L}\left(x_{i}\right)=\frac{1}{3}\left\{1-\left[\operatorname { m a x } \left(\left|M V_{A L}\left(x_{i}\right)-M V_{B L}\left(x_{i}\right)\right| \mathrm{V}\right.\right.\right. \\
& \left|M V_{A U}\left(x_{i}\right)-M V_{B U}\left(x_{i}\right)\right|,\left|N V_{A L}\left(x_{i}\right)-N V_{B L}\left(x_{i}\right)\right| \mathrm{\vee} \\
& \left|N V_{A U}\left(x_{i}\right)-N V_{B U}\left(x_{i}\right)\right|,\left|H V_{A L}\left(x_{i}\right)-H V_{B L}\left(x_{i}\right)\right| \mathrm{V} \\
& \left.\left.\left.\left|H V_{A U}\left(x_{i}\right)-H V_{B U}\left(x_{i}\right)\right|\right)\right]^{1 / 2}\right\} ; \\
& M V_{\emptyset(A, B) U}\left(x_{i}\right)=\frac{1}{3}\left\{1-\left[\operatorname { m a x } \left(\left|M V_{A L}\left(x_{i}\right)-M V_{B L}\left(x_{i}\right)\right| \mathrm{V}\right.\right.\right. \\
& \left|M V_{A U}\left(x_{i}\right)-M V_{B U}\left(x_{i}\right)\right|,\left|N V_{A L}\left(x_{i}\right)-N V_{B L}\left(x_{i}\right)\right| \mathrm{V} \\
& \left|N_{A U}\left(x_{i}\right)-N_{B U}\left(x_{i}\right)\right|,\left|H_{A L}\left(x_{i}\right)-H_{B L}\left(x_{i}\right)\right| \mathrm{V} \\
& \left.\left.\left.\left|H_{A U}\left(x_{i}\right)-H_{B U}\left(x_{i}\right)\right|\right)\right]\right\} ; \\
& N V_{\varnothing(A, B) L}\left(x_{i}\right)=\frac{1}{3}\left\{1+\left[\operatorname { m i n } \left(\left|M V_{A L}\left(x_{i}\right)-M V_{B L}\left(x_{i}\right)\right| \mathrm{V}\right.\right.\right. \\
& \left|M V_{A U}\left(x_{i}\right)-M V_{B U}\left(x_{i}\right)\right|,\left|N V_{A L}\left(x_{i}\right)-N V_{B L}\left(x_{i}\right)\right| \mathrm{V}
\end{aligned}
$$


$\left|N V_{A U}\left(x_{i}\right)-N V_{B U}\left(x_{i}\right)\right|,\left|H V_{A L}\left(x_{i}\right)-H V_{B L}\left(x_{i}\right)\right| \mathrm{V}$ $\left.\left.\left.\left|H V_{A U}\left(x_{i}\right)-H V_{B U}\left(x_{i}\right)\right|\right)\right]^{2}\right\}$;

$N V_{\varnothing(A, B) U}\left(x_{i}\right)=\frac{1}{3}\left\{1+\left[\min \left(\left|M V_{A L}\left(x_{i}\right)-M V_{B L}\left(x_{i}\right)\right| \mathrm{V}\right.\right.\right.$

$\left|M V_{A U}\left(x_{i}\right)-M V_{B U}\left(x_{i}\right)\right|,\left|N V_{A L}\left(x_{i}\right)-N V_{B L}\left(x_{i}\right)\right| \mathrm{V}$

$\left|N V_{A U}\left(x_{i}\right)-N V_{B U}\left(x_{i}\right)\right|,\left|H V_{A L}\left(x_{i}\right)-H V_{B L}\left(x_{i}\right)\right| \vee$

$\left.\left.\left.\left|H V_{A U}\left(x_{i}\right)-H V_{B U}\left(x_{i}\right)\right|\right)\right]\right\}$.

Theorem 4: $\mathrm{E}(\varnothing(\mathrm{A}, \mathrm{B}))$ be a similarity measure for IvIFSs Aand B, where $\mathrm{E}$ is an entropy.

Proof: To prove that $E(\varnothing(A, B))$ is a measure of similarity, we need to prove property given by definition 3 holds

Property 1): If $A \in \mathbb{C}(\Omega) \Rightarrow A\left(x_{i}\right)=\langle[1,1],[0,0],[0,0]\rangle$ or $A\left(x_{i}\right)=\langle[0,0],[1,1],[0,0]\rangle$, for any $x_{i} \in \Omega$, then $M V_{\emptyset(A, \bar{A}) L}\left(x_{i}\right)=0=M V_{\emptyset(A, \bar{A}) U}\left(x_{i}\right)$;

And $N V_{\emptyset(A, \bar{A}) L}\left(x_{i}\right)=1=N V_{\emptyset(A, \bar{A}) U}\left(x_{i}\right)$

Thus, $\emptyset(A, \bar{A})=\left\{\left\langle x_{i},[0,0],[1,1],[0,0]\right\rangle / x_{i} \in \Omega\right\}$

$\Longrightarrow \mathrm{S}(\mathrm{A}, \overline{\mathrm{A}})=\mathrm{E}(\varnothing(\mathrm{A}, \overline{\mathrm{A}}))=0$

Property 2): Assume that $S(A, B)=1 \Rightarrow E(\varnothing(A, B))=1$ $\Leftrightarrow M V_{\emptyset(A, B)}\left(x_{i}\right)=N V_{\emptyset(A, B)}\left(x_{i}\right)=H V_{\emptyset(A, B)}\left(x_{i}\right)=\left[\frac{1}{3}, \frac{1}{3}\right]$ $\Leftrightarrow \max \left(\left|M V_{A L}\left(x_{i}\right)-M V_{B L}\left(x_{i}\right)\right|\right.$

$$
\mathrm{V}\left|M V_{A U}\left(x_{i}\right)-M V_{B U}\left(x_{i}\right)\right|, \mid N V_{A L}\left(x_{i}\right)
$$$$
-N V_{B L}\left(x_{i}\right) \mid
$$$$
\mathrm{\vee}\left|N V_{A U}\left(x_{i}\right)-N V_{B U}\left(x_{i}\right)\right|, \mid H V_{A L}\left(x_{i}\right)
$$$$
-H V_{B L}\left(x_{i}\right) \mid
$$$$
\left.\mathrm{\vee}\left|H V_{A U}\left(x_{i}\right)-H V_{B U}\left(x_{i}\right)\right|\right)=0
$$

and $\min \left(\left|M V_{A L}\left(x_{i}\right)-M V_{B L}\left(x_{i}\right)\right| \vee \mid M V_{A U}\left(x_{i}\right)-\right.$ $M V_{B U}\left(x_{i}\right)||, N V_{A L}\left(x_{i}\right)-N V_{B L}\left(x_{i}\right)|\vee| N V_{A U}\left(x_{i}\right)-$ $N V_{B U}\left(x_{i}\right)||, H V_{A L}\left(x_{i}\right)-H V_{B L}\left(x_{i}\right)|\vee| H V_{A U}\left(x_{i}\right)-$ $\left.H V_{B U}\left(x_{i}\right) \mid\right)=0$

$\Leftrightarrow\left|M V_{A L}\left(x_{i}\right)-M V_{B L}\left(x_{i}\right)\right| \vee\left|M V_{A U}\left(x_{i}\right)-M V_{B U}\left(x_{i}\right)\right|$ $=0$,

$\left|N V_{A L}\left(x_{i}\right)-N V_{B L}\left(x_{i}\right)\right| \vee\left|N V_{A U}\left(x_{i}\right)-N V_{B U}\left(x_{i}\right)\right|=0$, and $\left|H V_{A L}\left(x_{i}\right)-H V_{B L}\left(x_{i}\right)\right| \vee\left|H V_{A U}\left(x_{i}\right)-H V_{B U}\left(x_{i}\right)\right|=$ 0 .

$$
\begin{aligned}
& \Leftrightarrow M V_{A L}\left(x_{i}\right)=M V_{B L}\left(x_{i}\right), M V_{A U}\left(x_{i}\right)=M V_{B U}\left(x_{i}\right), \\
& N V_{A L}\left(x_{i}\right)=N V_{B L}\left(x_{i}\right), N V_{A U}\left(x_{i}\right)=N V_{B U}\left(x_{i}\right) \\
& \quad \text { and } H V_{A L}\left(x_{i}\right)=H V_{B L}\left(x_{i}\right), H V_{A U}\left(x_{i}\right)=H V_{B U}\left(x_{i}\right) . \\
& \Leftrightarrow \mathrm{A}=\mathrm{B} .
\end{aligned}
$$

Property 3): $\varnothing(A, B)=\emptyset(B, A)$ by definition of $M V_{\emptyset(A, B) L}\left(x_{i}\right), M V_{\emptyset(A, B) U}\left(x_{i}\right), N V_{\emptyset(A, B) L}\left(x_{i}\right)$,

$N V_{\emptyset(A, B) U}\left(x_{i}\right)$ for any $x_{i} \in \Omega$

$$
\begin{gathered}
\Rightarrow E(\varnothing(A, B))=E(\varnothing(B, A)) \\
\Leftrightarrow S(A, B)=S(B, A)
\end{gathered}
$$

Property 4): Let A, B and C be any three IvIFSs such that $\mathrm{A} \subseteq \mathrm{B} \subseteq \mathrm{C}$ for any $x_{i} \in \Omega$, we have $M V_{A}\left(x_{i}\right) \leq$ $M V_{B}\left(x_{i}\right) \leq M V_{C}\left(x_{i}\right), N V_{A}\left(x_{i}\right) \geq N V_{B}\left(x_{i}\right) \geq N V_{C}\left(x_{i}\right)$ or $M V_{A L}\left(x_{i}\right) \leq M V_{B L}\left(x_{i}\right) \leq M V_{C L}\left(x_{i}\right), N V_{A L}\left(x_{i}\right) \geq$ $N V_{B L}\left(x_{i}\right) \geq N V_{C L}\left(x_{i}\right)$ and $M V_{A U}\left(x_{i}\right) \leq M V_{B U}\left(x_{i}\right) \leq$ $M V_{C U}\left(x_{i}\right), N V_{A U}\left(x_{i}\right) \geq N V_{B U}\left(x_{i}\right) \geq N V_{C U}\left(x_{i}\right)$. $\Rightarrow\left|M V_{A L}\left(x_{i}\right)-M V_{C L}\left(x_{i}\right)\right| \geq\left|M V_{A L}\left(x_{i}\right)-M V_{B L}\left(x_{i}\right)\right|$, $\left|M V_{A U}\left(x_{i}\right)-M V_{C U}\left(x_{i}\right)\right| \geq\left|M V_{A U}\left(x_{i}\right)-M V_{B U}\left(x_{i}\right)\right|$;
$\left|N V_{A L}\left(x_{i}\right)-N V_{C L}\left(x_{i}\right)\right| \geq\left|N V_{A L}\left(x_{i}\right)-N V_{B L}\left(x_{i}\right)\right|$, $\left|N V_{A U}\left(x_{i}\right)-N V_{C U}\left(x_{i}\right)\right| \geq\left|N V_{A U}\left(x_{i}\right)-N V_{B U}\left(x_{i}\right)\right| ;$ and $\quad\left|H V_{A L}\left(x_{i}\right)-H V_{C L}\left(x_{i}\right)\right|=\mid 2\left(M V_{C L}\left(x_{i}\right)-\right.$ $\left.M V_{A L}\left(x_{i}\right)\right)+2\left(N V_{C L}\left(x_{i}\right)-N V_{A L}\left(x_{i}\right)\right) \mid \geq$ $\mid 2\left(M V_{B L}\left(x_{i}\right)-M V_{A L}\left(x_{i}\right)\right)+2\left(N V_{B L}\left(x_{i}\right)-\right.$ $\left.N V_{A L}\left(x_{i}\right)\right)|=| N V_{A L}\left(x_{i}\right)-N V_{B L}\left(x_{i}\right) \mid$,

Similarly, we have $\left|N V_{A U}\left(x_{i}\right)-N V_{C U}\left(x_{i}\right)\right| \geq$ $\left|N V_{A U}\left(x_{i}\right)-N V_{B U}\left(x_{i}\right)\right|$

So, we have

$M V_{\emptyset(A, B)}\left(x_{i}\right) \leq M V_{\emptyset(A, C)}\left(x_{i}\right) \leq\left[\frac{1}{3}, \frac{1}{3}\right]$ and $N V_{\emptyset(A, B)}\left(x_{i}\right) \geq$ $N V_{\emptyset(A, C)}\left(x_{i}\right) \geq\left[\frac{1}{3}, \frac{1}{3}\right]$ for any $x_{i} \in \Omega . \Rightarrow \varnothing(\mathrm{A}, \mathrm{C}) \subseteq$ $\emptyset(\mathrm{A}, \mathrm{B}) \subseteq\left\langle\left[\frac{1}{3}, \frac{1}{3}\right],\left[\frac{1}{3}, \frac{1}{3}\right],\left[\frac{1}{3}, \frac{1}{3}\right]\right\rangle$

Similarly, we have $\Rightarrow \varnothing(\mathrm{A}, \mathrm{C}) \subseteq \varnothing(\mathrm{B}, \mathrm{C}) \subseteq$ $\left\langle\left[\frac{1}{3}, \frac{1}{3}\right],\left[\frac{1}{3}, \frac{1}{3}\right],\left[\frac{1}{3}, \frac{1}{3}\right]\right\rangle$.

$\mathrm{D}\left(\emptyset(\mathrm{A}, \mathrm{B}),\left\langle\left[\frac{1}{3}, \frac{1}{3}\right],\left[\frac{1}{3}, \frac{1}{3}\right],\left[\frac{1}{3}, \frac{1}{3}\right]\right\rangle\right) \leq$

$\mathrm{D}\left(\varnothing(\mathrm{A}, \mathrm{C}),\left\langle\left[\frac{1}{3}, \frac{1}{3}\right],\left[\frac{1}{3}, \frac{1}{3}\right],\left[\frac{1}{3}, \frac{1}{3}\right]\right\rangle\right)$

and $\quad \mathrm{D}\left(\varnothing(\mathrm{B}, \mathrm{C}),\left\langle\left[\frac{1}{3}, \frac{1}{3}\right],\left[\frac{1}{3}, \frac{1}{3}\right],\left[\frac{1}{3}, \frac{1}{3}\right]\right\rangle\right) \leq$ $\mathrm{D}\left(\varnothing(\mathrm{A}, \mathrm{C}),\left\langle\left[\frac{1}{3}, \frac{1}{3}\right],\left[\frac{1}{3}, \frac{1}{3}\right],\left[\frac{1}{3}, \frac{1}{3}\right]\right\rangle\right)$.

So form definition of entropy corresponding to distance function, we get $\mathrm{E}(\varnothing(\mathrm{A}, \mathrm{C})) \leq \mathrm{E}(\varnothing(\mathrm{A}, \mathrm{B}))$ and $\mathrm{E}(\varnothing(\mathrm{A}, \mathrm{C})) \leq \mathrm{E}(\varnothing(\mathrm{B}, \mathrm{C}))$ or $S(\varnothing(A, C)) \leq S(\varnothing(A, B)) \quad$ and $\quad S(\varnothing(A, C)) \leq$ $S(\varnothing(B, C))$.

Corollary 1: Let $\mathrm{E}$ be an entropy measure for IvIFSs and $\varnothing(A, B)$ be an IvIFS defined on two IvIFSs $A$ and $B$ acaccording to definition 6 , then $\mathrm{E}(\overline{\varnothing(\mathrm{A}, \mathrm{B})})$ measure of similarity for $A, B \in \operatorname{IvIFSS}(\Omega)$.

Proof: Proof followed from the definition of complement interval-valued intuitionistic fuzzy sets and theorem 4.

Definition 7: Let $A, B \in \operatorname{IvIFSS}(\Omega)$, we can define IvIFS $\eta(\mathrm{A}, \mathrm{B})$ using $\mathrm{A}, \mathrm{B}$ as follows:

$M V_{\eta(A, B) L}\left(x_{i}\right)=\frac{1}{3}\left\{1+\left[\min \left(\left(\left|M V_{A L}\left(x_{i}\right)-M V_{B L}\left(x_{i}\right)\right| \mathrm{V}\right.\right.\right.\right.$ $\left.\left|M V_{A U}\left(x_{i}\right)-M V_{B U}\left(x_{i}\right)\right|^{\alpha}\right),\left(\left|N V_{A L}\left(x_{i}\right)-N V_{B L}\left(x_{i}\right)\right| \mathrm{V}\right.$ $\left.\left|N V_{A U}\left(x_{i}\right)-N V_{B U}\left(x_{i}\right)\right|\right)^{\alpha},\left(\left|H V_{A L}\left(x_{i}\right)-H V_{B L}\left(x_{i}\right)\right| \vee\right.$ $\left.\left.\left.\left.\left|H V_{A U}\left(x_{i}\right)-H V_{B U}\left(x_{i}\right)\right|\right)^{\alpha}\right)\right]^{2}\right\}$;

$M V_{\eta(A, B) U}\left(x_{i}\right)=\frac{1}{3}\left\{1+\left[\min \left(\left(\left|M V_{A L}\left(x_{i}\right)-M V_{B L}\left(x_{i}\right)\right| \mathrm{V}\right.\right.\right.\right.$ $\left.\left|M V_{A U}\left(x_{i}\right)-M V_{B U}\left(x_{i}\right)\right|^{\alpha}\right),\left(\left|N V_{A L}\left(x_{i}\right)-N V_{B L}\left(x_{i}\right)\right| \mathrm{V}\right.$ $\left.\left|N V_{A U}\left(x_{i}\right)-N V_{B U}\left(x_{i}\right)\right|\right)^{\alpha},\left(\left|H V_{A L}\left(x_{i}\right)-H V_{B L}\left(x_{i}\right)\right| \mathrm{V}\right.$ $\left.\left.\left.\left.\left|H V_{A U}\left(x_{i}\right)-H V_{B U}\left(x_{i}\right)\right|\right)^{\alpha}\right)\right]\right\}$;

$N V_{\eta(A, B) L}\left(x_{i}\right)=\frac{1}{3}\left\{1-\left[\max \left(\left(\left|M V_{A L}\left(x_{i}\right)-M V_{B L}\left(x_{i}\right)\right| \mathrm{V}\right.\right.\right.\right.$ $\left.\left|M V_{A U}\left(x_{i}\right)-M V_{B U}\left(x_{i}\right)\right|^{\alpha}\right),\left(\left|N V_{A L}\left(x_{i}\right)-N V_{B L}\left(x_{i}\right)\right| \mathrm{V}\right.$ $\left.\left|N V_{A U}\left(x_{i}\right)-N V_{B U}\left(x_{i}\right)\right|\right)^{\alpha},\left(\left|H V_{A L}\left(x_{i}\right)-H V_{B L}\left(x_{i}\right)\right| \vee\right.$ $\left.\left.\left.\left.\left|H V_{A U}\left(x_{i}\right)-H V_{B U}\left(x_{i}\right)\right|\right)^{\alpha}\right)\right]^{1 / 2}\right\}$;

$N V_{\eta(A, B) U}\left(x_{i}\right)=\frac{1}{3}\left\{1-\left[\max \left(\left(\left|M V_{A L}\left(x_{i}\right)-M V_{B L}\left(x_{i}\right)\right| \mathrm{V}\right.\right.\right.\right.$ $\left.\left|M V_{A U}\left(x_{i}\right)-M V_{B U}\left(x_{i}\right)\right|^{\alpha}\right),\left(\left|N V_{A L}\left(x_{i}\right)-N V_{B L}\left(x_{i}\right)\right| \mathrm{V}\right.$ $\left.\left|N V_{A U}\left(x_{i}\right)-N V_{B U}\left(x_{i}\right)\right|\right)^{\alpha},\left(\left|H V_{A L}\left(x_{i}\right)-H V_{B L}\left(x_{i}\right)\right| \vee\right.$ $\left.\left.\left.\left.\left|H V_{A U}\left(x_{i}\right)-H V_{B U}\left(x_{i}\right)\right|\right)^{\alpha}\right)\right]\right\}$

Where $\alpha \in\left[1, \infty\left[\right.\right.$ and $x_{i} \in \Omega$. 
Theorem 5: For any two IvIFSs A and B, $\mathrm{E}(\eta(\mathrm{A}, \mathrm{B}))$ is a measure of similarity, where $\mathrm{E}$ is an entropy measure.

Proof: To prove that $E(\eta(A, B))$ is a measure of similarity, we need to prove property given by definition 3 holds .

Property 1): If $A \in \mathbb{C}(\Omega) \Rightarrow A\left(x_{i}\right)=\langle[1,1],[0,0],[0,0]\rangle$ or $A\left(x_{i}\right)=\langle[0,0],[1,1],[0,0]\rangle$, for any $x_{i} \in \Omega$, then $M V_{\emptyset(A, \bar{A}) L}\left(x_{i}\right)=1=M V_{\emptyset(A, \bar{A}) U}\left(x_{i}\right)$;

and $N V_{\emptyset(A, \bar{A}) L}\left(x_{i}\right)=0=N V_{\emptyset(A, \bar{A}) U}\left(x_{i}\right)$

Thus, $\emptyset(\mathrm{A}, \overline{\mathrm{A}})=\left\{\left\langle x_{i},[1,1],[0,0],[0,0]\right\rangle / x_{i} \in \Omega\right\}$

$\Rightarrow \mathrm{S}(\mathrm{A}, \overline{\mathrm{A}})=\mathrm{E}(\varnothing(\mathrm{A}, \overline{\mathrm{A}}))=0$

Property 2): Assume that $\mathrm{S}(\mathrm{A}, \mathrm{B})=1$

Then $\mathrm{E}(\eta(\mathrm{A}, \mathrm{B}))=1$

$\Leftrightarrow M V_{\eta(A, B)}\left(x_{i}\right)=\left[\frac{1}{3}, \frac{1}{3}\right]=N V_{\eta(A, B)}\left(x_{i}\right)$

$=H V_{\eta(A, B)}\left(x_{i}\right)$

$\Leftrightarrow \min \left(\begin{array}{c}\left(\left|M V_{A L}\left(x_{i}\right)-M V_{B L}\left(x_{i}\right)\right| \vee\left|M V_{A U}\left(x_{i}\right)-M V_{B U}\left(x_{i}\right)\right|\right)^{a} \\ \left(\left|N V_{A L}\left(x_{i}\right)-N V_{B L}\left(x_{i}\right)\right| \vee\left|N V_{A U}\left(x_{i}\right)-N V_{B U}\left(x_{i}\right)\right|\right)^{\alpha} \\ \left(\left|H V_{A L}\left(x_{i}\right)-H V_{B L}\left(x_{i}\right)\right| \vee\left|H V_{A U}\left(x_{i}\right)-H V_{B U}\left(x_{i}\right)\right|\right)^{\alpha}\end{array}\right.$ $=0$

and

$\max \left(\begin{array}{c}\left(\left|M V_{A L}\left(x_{i}\right)-M V_{B L}\left(x_{i}\right)\right| \vee\left|M V_{A U}\left(x_{i}\right)-M V_{B U}\left(x_{i}\right)\right|\right)^{\alpha}, \\ \left(\left|N V_{A L}\left(x_{i}\right)-N V_{B L}\left(x_{i}\right)\right| \vee\left|N V_{A U}\left(x_{i}\right)-N V_{B U}\left(x_{i}\right)\right|\right)^{\alpha}, \\ \left(\left|H V_{A L}\left(x_{i}\right)-H V_{B L}\left(x_{i}\right)\right| \vee\left|H V_{A U}\left(x_{i}\right)-H V_{B U}\left(x_{i}\right)\right|\right)^{\alpha}\end{array}\right)$ $=0$

$$
\begin{aligned}
& \Leftrightarrow\left(\left|M V_{A L}\left(x_{i}\right)-M V_{B L}\left(x_{i}\right)\right|\right. \\
& \left.\mathrm{V}\left|M V_{A U}\left(x_{i}\right)-M V_{B U}\left(x_{i}\right)\right|\right)^{\alpha}=0 \text {, } \\
& \left(\left|N V_{A L}\left(x_{i}\right)-N V_{B L}\left(x_{i}\right)\right| \vee\left|N V_{A U}\left(x_{i}\right)-N V_{B U}\left(x_{i}\right)\right|\right)^{\alpha} \\
& =0 \text {, } \\
& \text { and } \quad\left(\left|H V_{A L}\left(x_{i}\right)-H V_{B L}\left(x_{i}\right)\right| \vee \mid H V_{A U}\left(x_{i}\right)-\right. \\
& \left.H V_{B U}\left(x_{i}\right) \mid\right)^{\alpha}=0 \text {. } \\
& \Leftrightarrow M V_{A L}\left(x_{i}\right)=M V_{B L}\left(x_{i}\right), M V_{A U}\left(x_{i}\right) \\
& =M V_{B U}\left(x_{i}\right), N V_{A L}\left(x_{i}\right) \\
& =N V_{B L}\left(x_{i}\right), N V_{A U}\left(x_{i}\right)=N V_{B U}\left(x_{i}\right)
\end{aligned}
$$

and $H V_{A L}\left(x_{i}\right)=H V_{B L}\left(x_{i}\right), H V_{A U}\left(x_{i}\right)=H V_{B U}\left(x_{i}\right)$.

$$
\Leftrightarrow \mathrm{A}=\mathrm{B} \text {. }
$$

Property 3): $\eta(\mathrm{A}, \mathrm{B})=\eta(\mathrm{B}, \mathrm{A})$ by definition of $M V_{\eta(A, B) L}\left(x_{i}\right), M V_{\eta(A, B) U}\left(x_{i}\right), N V_{\eta(A, B) L}\left(x_{i}\right)$,

$N V_{\eta(A, B) U}\left(x_{i}\right)$ for any $x_{i} \in \Omega$

$\Rightarrow \mathrm{E}(\varnothing(\mathrm{A}, \mathrm{B}))=\mathrm{E}(\varnothing(\mathrm{B}, \mathrm{A}))$

$\Leftrightarrow \mathrm{S}(\mathrm{A}, \mathrm{B})=\mathrm{S}(\mathrm{B}, \mathrm{A})$

Property 4): Let A, B and C be any three IvIFSs such that $\mathrm{A} \subseteq \mathrm{B} \subseteq \mathrm{C}$, then for any $x_{i} \in \Omega$, we have $M V_{A}\left(x_{i}\right) \leq$ $M V_{B}\left(x_{i}\right) \leq M V_{C}\left(x_{i}\right), N V_{A}\left(x_{i}\right) \geq N V_{B}\left(x_{i}\right) \geq N V_{C}\left(x_{i}\right)$ or $M V_{A L}\left(x_{i}\right) \leq M V_{B L}\left(x_{i}\right) \leq M V_{C L}\left(x_{i}\right), N V_{A L}\left(x_{i}\right) \geq$

$N V_{B L}\left(x_{i}\right) \geq N V_{C L}\left(x_{i}\right) \quad$ and $M V_{A U}\left(x_{i}\right) \leq M V_{B U}\left(x_{i}\right) \leq$

$M V_{C U}\left(x_{i}\right), N V_{A U}\left(x_{i}\right) \geq N V_{B U}\left(x_{i}\right) \geq N V_{C U}\left(x_{i}\right)$.

$\Rightarrow\left|M V_{A L}\left(x_{i}\right)-M V_{C L}\left(x_{i}\right)\right| \geq\left|M V_{A L}\left(x_{i}\right)-M V_{B L}\left(x_{i}\right)\right|$,

$\left|M V_{A U}\left(x_{i}\right)-M V_{C U}\left(x_{i}\right)\right| \geq\left|M V_{A U}\left(x_{i}\right)-M V_{B U}\left(x_{i}\right)\right|$;

$\left|N V_{A L}\left(x_{i}\right)-N V_{C L}\left(x_{i}\right)\right| \geq\left|N V_{A L}\left(x_{i}\right)-N V_{B L}\left(x_{i}\right)\right|$,

$\left|N V_{A U}\left(x_{i}\right)-N V_{C U}\left(x_{i}\right)\right| \geq\left|N V_{A U}\left(x_{i}\right)-N V_{B U}\left(x_{i}\right)\right|$; and $\quad\left|H V_{A L}\left(x_{i}\right)-H V_{C L}\left(x_{i}\right)\right|=\mid 2\left(M V_{C L}\left(x_{i}\right)-\right.$ $\left.M V_{A L}\left(x_{i}\right)\right)+2\left(N V_{C L}\left(x_{i}\right)-N V_{A L}\left(x_{i}\right)\right) \mid \geq$

$\mid 2\left(M V_{B L}\left(x_{i}\right)-M V_{A L}\left(x_{i}\right)\right)+2\left(N V_{B L}\left(x_{i}\right)-\right.$

$\left.N V_{A L}\left(x_{i}\right)\right)|=| N V_{A L}\left(x_{i}\right)-N V_{B L}\left(x_{i}\right) \mid$,

Similarly, we have $\left|N V_{A U}\left(x_{i}\right)-N V_{C U}\left(x_{i}\right)\right| \geq$ $\left|N V_{A U}\left(x_{i}\right)-N V_{B U}\left(x_{i}\right)\right|$

So, we have from definition 7

$M V_{\eta(A, C)}\left(x_{i}\right) \geq M V_{\eta(A, B)}\left(x_{i}\right) \geq\left[\frac{1}{3}, \frac{1}{3}\right]$ and $N V_{\eta(A, C)}\left(x_{i}\right) \leq$ $N V_{\eta(A, B)}\left(x_{i}\right) \leq\left[\frac{1}{3}, \frac{1}{3}\right]$ for any $x_{i} \in \Omega . \Rightarrow \eta(\mathrm{A}, \mathrm{C}) \supseteq$ $\eta(\mathrm{A}, \mathrm{B}) \supseteq\left\langle\left[\frac{1}{3}, \frac{1}{3}\right],\left[\frac{1}{3}, \frac{1}{3}\right],\left[\frac{1}{3}, \frac{1}{3}\right]\right\rangle$

Similarly, we have $\Rightarrow \eta(\mathrm{A}, \mathrm{C}) \supseteq \eta(\mathrm{B}, \mathrm{C}) \supseteq$ $\left\langle\left[\frac{1}{3}, \frac{1}{3}\right],\left[\frac{1}{3}, \frac{1}{3}\right],\left[\frac{1}{3}, \frac{1}{3}\right]\right\rangle$. Thus

$\mathrm{D}\left(\eta(\mathrm{A}, \mathrm{B}),\left\langle\left[\frac{1}{3}, \frac{1}{3}\right],\left[\frac{1}{3}, \frac{1}{3}\right],\left[\frac{1}{3}, \frac{1}{3}\right]\right\rangle\right)$

$$
\leq \mathrm{D}\left(\eta(\mathrm{A}, \mathrm{C}),\left\langle\left[\frac{1}{3}, \frac{1}{3}\right],\left[\frac{1}{3}, \frac{1}{3}\right],\left[\frac{1}{3}, \frac{1}{3}\right]\right\rangle\right)
$$

and $\mathrm{D}\left(\eta(\mathrm{B}, \mathrm{C}),\left\langle\left[\frac{1}{3}, \frac{1}{3}\right],\left[\frac{1}{3}, \frac{1}{3}\right],\left[\frac{1}{3}, \frac{1}{3}\right]\right\rangle\right) \leq$

$\mathrm{D}\left(\eta(\mathrm{A}, \mathrm{C}),\left\langle\left[\frac{1}{3}, \frac{1}{3}\right],\left[\frac{1}{3}, \frac{1}{3}\right],\left[\frac{1}{3}, \frac{1}{3}\right]\right\rangle\right)$.

So from definition of entropy corresponding to distance function, we get $\mathrm{E}(\varnothing(\mathrm{A}, \mathrm{C})) \leq \mathrm{E}(\varnothing(\mathrm{A}, \mathrm{B}))$ and $\mathrm{E}(\eta(\mathrm{A}, \mathrm{C})) \leq \mathrm{E}(\eta(\mathrm{B}, \mathrm{C}))$

or $S(\eta(A, C)) \leq S(\eta(A, B)) \quad$ and $\quad S(\eta(A, C)) \leq$ $S(\eta(B, C))$.

Corollary 2: Let $\mathrm{E}$ be an entropy for IvIFSs and $\eta(\mathrm{A}, \mathrm{B})$ be an IVIFS defined on $A, B \in \operatorname{IvIFSs}(\Omega)$ as defined in definition 7 , then $\mathrm{E}(\overline{\eta(\mathrm{A}, \mathrm{B})})$ is measure of similarity for $A, B \in \operatorname{IvIFSs}(\Omega)$.

Proof: Proof followed from the definition of complement of IVIFSS and theorem 5.

\subsection{Weighted similarity measure}

Let $\mathrm{w}=\left(\mathrm{w}_{1}, \mathrm{w}_{2}, \ldots, \mathrm{w}_{\mathrm{n}}\right)^{\mathrm{T}}$ be the weights provided to each element $x_{i} \in \Omega, i=1,2, \ldots, n$. Then the weighted similarity measure based on the aforesaid similarity measures are defined as $S(A, B)=$ $\sum_{i=1}^{n} w_{i} S\left(A\left(x_{i}\right), B\left(x_{i}\right)\right)$, where $w_{i} \geq 0$ and $\sum_{i=1}^{n} w_{i}=1$.

\subsection{Comparison with some select measures of similarity}

Here, we compare the performance of proposed measure of similarity with some of the existing similarity measures as follows.

For any two IvIFSs A and B, then some existing similarity measures are given as follows:

- $S_{W}(A, B)=$

$\frac{1}{-} \sum_{i=1}^{n} \frac{4-\left(M V_{L}\left(x_{i}\right)+M V_{U}\left(x_{i}\right)+N V_{L}\left(x_{i}\right)+N V_{U}\left(x_{i}\right)\right)+\left(H V_{L}\left(x_{i}\right)+H V_{U}\left(x_{i}\right)\right)}{4+\left(M V_{L}\left(x_{i}\right)+M V_{U}\left(x_{i}\right)+N V_{L}\left(x_{i}\right)+N V_{U}\left(x_{i}\right)\right)+\left(H V_{L}\left(x_{i}\right)+H V_{U}\left(x_{i}\right)\right)}$ $\frac{1}{n} \sum_{i=1}^{n} \frac{\left(M V_{L}\left(x_{i}\right)+M V_{U}\left(x_{i}\right)+N V_{L}\left(x_{i}\right)+N V_{U}\left(x_{i}\right)\right)+\left(H V_{L}\left(x_{i}\right)+H V_{U}\left(x_{i}\right)\right)}{4+\left(x_{i}\right.}$

where $M V_{L}\left(x_{i}\right)=\left|M V_{A L}\left(x_{i}\right)-M V_{B L}\left(x_{i}\right)\right|, \quad M V_{U}\left(x_{i}\right)=$ $\left|M V_{A U}\left(x_{i}\right)-M V_{B U}\left(x_{i}\right)\right|, \quad N V_{L}\left(x_{i}\right)=\mid N V_{A L}\left(x_{i}\right)-$ $N V_{B L}\left(x_{i}\right)\left|, \quad N V_{U}\left(x_{i}\right)=\right| N V_{A U}\left(x_{i}\right)-$ $N V_{B U}\left(x_{i}\right) \mid, H V_{L}\left(x_{i}\right)=H V_{A L}\left(x_{i}\right)+H V_{B L}\left(x_{i}\right) \quad$ and $H V_{U}\left(x_{i}\right)=H V_{A U}\left(x_{i}\right)+H V_{B U}\left(x_{i}\right)$ is proposed by $\mathrm{Wu}$ et al.(2014). 
- $S_{H L}(A, B)=1-\frac{1}{4 n} \sum_{i=1}^{n} \mid M V_{A L}\left(x_{i}\right)-$ $M V_{B L}\left(x_{i}\right)|+| M V_{A U}\left(x_{i}\right)-M V_{B U}\left(x_{i}\right) \mid+$ $\left|N V_{A L}\left(x_{i}\right)-N V_{B L}\left(x_{i}\right)\right|+\left|N V_{A U}\left(x_{i}\right)-N V_{B U}\left(x_{i}\right)\right|$ is given by $\mathrm{Hu}$ and $\mathrm{Li}(2013)$.

- $S_{S}(A, B)=$

$\frac{1}{n} \sum_{i=1}^{n} \frac{\left[\begin{array}{l}\left(M V_{A L}\left(x_{i}\right)+M V_{A U}\left(x_{i}\right)\right)\left(M V_{B L}\left(x_{i}\right)+M V_{B U}\left(x_{i}\right)\right) \\ +\left(N V_{A L}\left(x_{i}\right)+N V_{A U}\left(x_{i}\right)\right)\left(N V_{B L}\left(x_{i}\right)+N V_{B U}\left(x_{i}\right)\right)\end{array}\right]}{\left[\begin{array}{l}\sqrt{\left(M V_{A L}\left(x_{i}\right)+M V_{A U}\left(x_{i}\right)\right)^{2}+\left(N V_{A L}\left(x_{i}\right)+N V_{A U}\left(x_{i}\right)\right)^{2}} \\ \sqrt{\left(M V_{B L}\left(x_{i}\right)+M V_{B U}\left(x_{i}\right)\right)^{2}+\left(N V_{B L}\left(x_{i}\right)+N V_{B U}\left(x_{i}\right)\right)^{2}}\end{array}\right]}$ is introduced by $\operatorname{Singh}(2012)$

- $S_{S u}(A, B)=$

$\left|M V_{A L}\left(x_{i}\right)-M V_{B L}\left(x_{i}\right)\right| \mathrm{V}\left|N V_{A L}\left(x_{i}\right)-N V_{B L}\left(x_{i}\right)\right|$ $\frac{1}{n} \sum_{i=1}^{n}+\left|M V_{A U}\left(x_{i}\right)-M V_{B U}\left(x_{i}\right)\right| \mathrm{v}\left|N V_{A U}\left(x_{i}\right)-N V_{B U}\left(x_{i}\right)\right|$

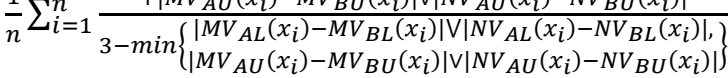

is proposed by Sun \& Liu (2012).

To review the performance of similarity measures let us consider an example. Consider the following IvIFSs $\mathrm{A}=\left\{x_{i},\langle[0.5,0.5],[0.5,0.5],[0,0]\rangle, x_{i} \in \Omega\right\}$, $\mathrm{B}=\left\{x_{i},\langle[0.3,0.4],[0.4,0.5],[0.1,0.3]\rangle, x_{i} \in \Omega\right\}$, $\mathrm{C}=\left\{x_{i},\langle[0.3,0.3],[0.3,0.3],[0.4,0.4]\rangle, x_{i} \in \Omega\right\}$, $\mathrm{D}=\left\{x_{i},\langle[0.6,0.6],[0.4,0.4],[0,0]\rangle, x_{i} \in \Omega\right\}$

Intuitively, it is clear that $\mathrm{A}$ is more similar to $\mathrm{D}$ than $\mathrm{B}$ and $\mathrm{C}$. The result corresponding to measure of similarity measures given in Table 3 :

\begin{tabular}{|c|l|l|l|}
\hline & \multicolumn{1}{|c|}{ A B } & \multicolumn{1}{|c|}{ A C } & \multicolumn{1}{c|}{ A D } \\
\hline $\mathrm{S}_{\mathrm{W}}$ & 0.83333 & 0.69308 & 0.8181 \\
\hline $\mathrm{S}_{\mathrm{HL}}$ & 0.9 & 0.8 & 0.9 \\
\hline $\mathrm{S}_{\mathrm{S}}$ & 0.99227 & 0.9 & 0.98058 \\
\hline $\mathrm{S}_{\mathrm{Su}}$ & 0.89655 & 0.8571 & 0.9310 \\
\hline $\mathrm{S}_{1}(\phi)$ & 0.7450 & 0.73722 & 0.8646 \\
\hline $\mathrm{S}_{2}(\phi)$ & 0.78806 & 0.761886 & 0.89594 \\
\hline $\mathrm{S}_{3}(\phi)$ & 0.72614 & 0.68377 & 0.84188 \\
\hline $\mathrm{S}_{4}(\phi)$ & 0.78806 & 0.74188 & 0.89594 \\
\hline $\mathrm{S}_{5}(\phi)$ & 0.68210 & 0.62283 & 0.84391 \\
\hline $\mathrm{S}_{6}(\phi)$ for $\mathrm{p}=2$ & 0.77639 & 0.77141 & 0.87090 \\
\hline $\mathrm{S}_{1}(\eta)$ & 0.93205 & 0.89426 & 0.98708 \\
\hline $\mathrm{S}_{2}(\eta)$ & 0.95217 & 0.92075 & 0.99184 \\
\hline $\mathrm{S}_{3}(\eta)$ & 0.91759 & 0.85853 & 0.98418 \\
\hline $\mathrm{S}_{4}(\eta)$ & 0.95217 & 0.92075 & 0.99184 \\
\hline $\mathrm{S}_{5}(\eta)$ & 0.92825 & 0.88113 & 0.98776 \\
\hline $\mathrm{S}_{6}(\eta)$ for $\mathrm{p}=2$ & 0.93292 & 0.89590 & 0.98709 \\
\hline
\end{tabular}

Table 3: Comparison of Similarity Measures.

From the similarity measures listed in table 3 , we can see that

$\mathrm{S}_{\mathrm{W}}, \mathrm{S}_{\mathrm{HL}}$ and $\mathrm{S}_{\mathrm{S}}$ are inconsistent with intuition where as $S_{S u}, S_{j}(\phi)$ and $S_{j}(\eta), j=1, \ldots, 6$.

In this section we have derived a relation between entropy and similarity measure. Then we defined some similarity measures, compared its performance with existing similarity measures. In section 5 we applied proposed similarity measures to draw conclusion in pattern recognition and medical diagnoses.

\section{Applications of proposed similarity measures}

Here the proposed similarity measures are applied to some of the situation that deals with imperfect information.

\subsection{Pattern recognition}

Here we use an example of pattern recognition considered by $\mathrm{Xu}$ (2007) and adapted by Wei et al. (2011) and Wu et al.(2014) for classification of building material.

Example: There are four types of building materials $A_{i}, i=1,2,3,4$ and an anonymous building material $\mathrm{B}$, which is characterized by the IvIFSs defined on $X=$ $\left\{x_{1}, x_{2}, \ldots, x_{12}\right\}$ with weighted vector

$$
\mathrm{w}=\left(\begin{array}{c}
0.1,0.05,0.08,0.06,0.03,0.07 \\
0.09,0.12,0.15,0.07,0.13,0.05
\end{array}\right)^{\mathrm{T}}
$$

and we have the data given as follows by $\mathrm{Xu}$ (2007).

$$
\left.\begin{array}{rl} 
& \text { ' } A_{1} \\
= & \left(\begin{array}{c}
\left\langle x_{1},[0.1,0.2],[0.5,0.6]\right\rangle,\left\langle x_{2},[0.1,0.2],[0.7,0.8]\right\rangle, \\
\left\langle x_{3},[0.5,0.6],[0.3,0.4]\right\rangle,\left\langle x_{4},[0.8,0.9],[0.0,0.1]\right\rangle,
\end{array}\right) \\
\left(\begin{array}{c}
\left\langle x_{5},[0.4,0.5],[0.3,0.4]\right\rangle,\left\langle x_{6},[0.0,0.1],[0.8,0.9]\right\rangle, \\
\left\langle x_{7},[0.3,0.4],[0.5,0.6]\right\rangle,\left\langle x_{8},[1.0,1.0],[0.0,0.0]\right\rangle,
\end{array}\right) \\
\left(\begin{array}{c}
\left\langle x_{9},[0.2,0.3],[0.6,0.7]\right\rangle,\left\langle x_{10},[0.4,0.5],[0.4,0.5]\right\rangle, \\
\left\langle x_{11},[0.7,0.8],[0.1,0.2]\right\rangle,\left\langle x_{12},[0.4,0.5],[0.4,0.5]\right\rangle
\end{array}\right)
\end{array}\right\}
$$

$A_{2}$

$$
=\left\{\begin{array}{c}
\left(\begin{array}{c}
\left\langle x_{1},[0.5,0.6],[0.3,0.4]\right\rangle,\left\langle x_{2},[0.6,0.7],[0.1,0.2]\right\rangle, \\
\left\langle x_{3},[1.0,1.0],[0.0,0.0]\right\rangle,\left\langle x_{4},[0.1,0.2],[0.6,0.7]\right\rangle,
\end{array}\right) \\
\left(\begin{array}{c}
\left\langle x_{5},[0.0,0.1],[0.8,0.9]\right\rangle,\left\langle x_{6},[0.7,0.8],[0.1,0.2]\right\rangle, \\
\left\langle x_{7},[0.5,0.6],[0.3,0.4]\right\rangle,\left\langle x_{8},[0.6,0.7],[0.2,0.3]\right\rangle,
\end{array}\right) \\
\left(\begin{array}{c}
\left\langle x_{9},[1.0,1.0],[0.0,0.0]\right\rangle,\left\langle x_{10},[0.1,0.2],[0.7,0.8]\right\rangle, \\
\left\langle x_{11},[0.0,0.1],[0.8,0.9]\right\rangle,\left\langle x_{12},[0.7,0.8],[0.1,0.2]\right\rangle
\end{array}\right)
\end{array}\right\}
$$

$A_{3}$

$$
=\left\{\begin{array}{c}
\left(\begin{array}{c}
\left\langle x_{1},[0.4,0.5],[0.3,0.4]\right\rangle,\left\langle x_{2},[0.6,0.7],[0.2,0.3]\right\rangle, \\
\left\langle x_{3},[0.9,1.0],[0.0,0.0]\right\rangle,\left\langle x_{4},[0.0,0.1],[0.8,0.9]\right\rangle,
\end{array}\right) \\
\left(\begin{array}{l}
\left\langle x_{5},[0.0,0.1],[0.8,0.9]\right\rangle,\left\langle x_{6},[0.6,0.7],[0.2,0.3]\right\rangle, \\
\left\langle x_{7},[0.1,0.2],[0.7,0.8]\right\rangle,\left\langle x_{8},[0.2,0.3],[0.6,0.7]\right\rangle,
\end{array}\right) \\
\left(\begin{array}{l}
\left\langle x_{9},[0.5,0.6],[0.2,0.4]\right\rangle,\left\langle x_{10},[1.0,1.0],[0.0,0.0]\right\rangle, \\
\left\langle x_{11},[0.3,0.4],[0.4,0.5]\right\rangle,\left\langle x_{12},[0.0,0.1],[0.8,0.9]\right\rangle
\end{array}\right)
\end{array}\right\}
$$

$A_{4}$ $=\left\{\begin{array}{c}\left(\begin{array}{c}\left\langle x_{1},[1.0,1.0],[0.0,0.0]\right\rangle,\left\langle x_{2},[1.0,1.0],[0.0,0.0]\right\rangle, \\ \left\langle x_{3},[0.8,0.9],[0.0,0.1]\right\rangle,\left\langle x_{4},[0.7,0.8],[0.1,0.2]\right\rangle,\end{array}\right) \\ \left(\begin{array}{l}\left\langle x_{5},[0.0,0.1],[0.7,0.9]\right\rangle,\left\langle x_{6},[0.0,0.1],[0.8,0.9]\right\rangle, \\ \left\langle x_{7},[0.1,0.2],[0.7,0.8]\right\rangle,\left\langle x_{8},[0.1,0.2],[0.7,0.8]\right\rangle\end{array}\right) \\ \left(\begin{array}{l}\left\langle x_{9},[0.4,0.5],[0.3,0.4]\right\rangle,\left\langle x_{10},[1.0,1.0],[0.0,0.0]\right\rangle, \\ \left\langle x_{11},[0.3,0.4],[0.4,0.5]\right\rangle,\left\langle x_{12},[0.0,0.1],[0.8,0.9]\right\rangle\end{array}\right)\end{array}\right\}$

$B=$

$\left\{\begin{array}{c}\left(\begin{array}{c}\left\langle x_{1},[0.9,1.0],[0.0,0.0]\right\rangle,\left\langle x_{2},[0.9,1.0],[0.0,0.0]\right\rangle, \\ \left\langle x_{3},[0.7,0.8],[0.1,0.2]\right\rangle,\left\langle x_{4},[0.6,0.7],[0.1,0.2]\right\rangle,\end{array}\right) \\ \left(\begin{array}{c}\left\langle x_{5},[0.0,0.1],[0.8,0.9]\right\rangle,\left\langle x_{6},[0.1,0.2],[0.7,0.8]\right\rangle_{,} \\ \left\langle x_{7},[0.1,0.2],[0.7,0.8]\right\rangle,\left\langle x_{8},[0.1,0.2],[0.7,0.8]\right\rangle,\end{array}\right) \\ \left(\begin{array}{c}\left\langle x_{9},[0.4,0.5],[0.3,0.4]\right\rangle,\left\langle x_{10},[1.0,1.0],[0.0,0.0]\right\rangle_{,} \\ \left\langle x_{11},[0.3,0.4],[0.4,0.5]\right\rangle,\left\langle x_{12},[0.0,0.1],[0.7,0.9]\right\rangle\end{array}\right)\end{array}\right\}$ 
We need to identify which pattern is most similar to B using the maximum degree principle of measures of similarity between IvIFSs. Using the anticipated similarity measures defined in this paper, we get the following results given in table 4 :

\begin{tabular}{|l|l|l|l|l|}
\hline & \multicolumn{1}{|c|}{$\mathrm{A}_{1} \mathrm{~B}$} & \multicolumn{1}{|c|}{$\mathrm{A}_{2} \mathrm{~B}$} & \multicolumn{1}{|c|}{$\mathrm{A}_{3} \mathrm{~B}$} & \multicolumn{1}{|c|}{$\mathrm{A}_{4} \mathrm{~B}$} \\
\hline $\mathrm{S}_{1}(\phi)$ & 0.688569 & 0.803061 & 0.84671 & 0.936548 \\
\hline $\mathrm{S}_{2}(\phi)$ & 0.725141 & 0.854747 & 0.868809 & 0.95075 \\
\hline $\mathrm{S}_{3}(\phi)$ & 0.654357 & 0.742627 & 0.818343 & 0.9265 \\
\hline $\mathrm{S}_{4}(\phi)$ & 0.725141 & 0.861997 & 0.868809 & 0.95075 \\
\hline $\mathrm{S}_{5}(\phi)$ & 0.587711 & 0.789371 & 0.803214 & 0.926125 \\
\hline $\begin{array}{l}\mathrm{S}_{6}(\phi) \\
\text { for } \mathrm{p}=2\end{array}$ & 0.738413 & 0.80768 & 0.863582 & 0.939988 \\
\hline $\mathrm{S}_{1}(\eta)$ & 0.780631 & 0.773645 & 0.768612 & 0.979413 \\
\hline $\mathrm{S}_{2}(\eta)$ & 0.816725 & 0.811175 & 0.804125 & 0.98595 \\
\hline $\mathrm{S}_{3}(\eta)$ & 0.767465 & 0.749444 & 0.76081 & 0.975 \\
\hline $\mathrm{S}_{4}(\eta)$ & 0.816725 & 0.811175 & 0.804125 & 0.98595 \\
\hline $\mathrm{S}_{5}(\eta)$ & 0.725088 & 0.716763 & 0.706188 & 0.978925 \\
\hline $\begin{array}{l}\mathrm{S}_{6}(\eta) \\
\text { for } \mathrm{p}=2\end{array}$ & 0.814325 & 0.801194 & 0.810194 & 0.979588 \\
\hline
\end{tabular}

Table 4: Application to pattern recognition.

From the above values it is clear that $B$ is most similar to $\mathrm{A}_{4}$ as the value corresponding to each similarity measure is highest for $\mathrm{A}_{4}$. So, we can conclude that $\mathrm{A}_{4}$ building material consistent with the specification and this result is consistent with the results presented by $\mathrm{Wu}$ et al.(2014).

\subsection{Medical diagnoses}

Many authors Wei et al. (2011), Wu et al. (2014), Singh (2012) employed IvIFSs to execute medical diagnosis in their works. Here we use the data used by Singh(2012) to do medical diagnosis using the proposed measure of similarity

Example: Let A and B be the set that represent the set of diagnoses and symptoms respectively given as $\mathrm{A}=$ $\left\{\left\langle\mathrm{A}_{1}\right.\right.$, Viral fever $\rangle,\left\langle\mathrm{A}_{2}\right.$, Malaria $\rangle,\left\langle\mathrm{A}_{3}\right.$, Typhoid $\left.\rangle\right\}$ and $=$ $\left\{\left\langle\mathrm{B}_{1}\right.\right.$, Temperature $\rangle,\left\langle\mathrm{B}_{2}\right.$, Headache $\rangle,\left\langle\mathrm{B}_{3}\right.$, Cough $\left.\rangle\right\}$

Assume the patient is represented by

$$
P=\left\{\begin{array}{c}
\left\langle B_{1},[0.6,0.8],[0.1,0.2]\right\rangle,\left\langle B_{2},[0.3,0.7],[0.2,0.3]\right\rangle, \\
\left\langle B_{3},[0.6,0.8],[0.1,0.2]\right\rangle
\end{array}\right\}
$$

and the weights corresponding to each attribute is equal and each diagnosis is given by the following IVIFSS

$$
\begin{aligned}
& A_{1}=\left\{\begin{array}{c}
\left\langle B_{1},[0.4,0.5],[0.3,0.4]\right\rangle,\left\langle B_{2},[0.4,0.6],[0.2,0.4]\right\rangle, \\
\left\langle B_{3},[0.4,0.8],[0.1,0.2]\right\rangle
\end{array}\right\} \\
& A_{2}=\left\{\begin{array}{c}
\left\langle B_{1},[0.3,0.6],[0.3,0.4]\right\rangle,\left\langle B_{2},[0.5,0.6],[0.3,0.4]\right\rangle, \\
\left\langle B_{3},[0.4,0.5],[0.1,0.3]\right\rangle
\end{array}\right\} \\
& A_{3}=\left\{\begin{array}{c}
\left\langle B_{1},[0.7,0.8],[0.1,0.2]\right\rangle,\left\langle B_{2},[0.6,0.7],[0.1,0.3]\right\rangle, \\
\left\langle B_{3},[0.3,0.4],[0.1,0.2]\right\rangle
\end{array}\right\}
\end{aligned}
$$

Using the proposed similarity measure we classify the patient $P$ in one of the diagnoses $A_{1}, A_{2}, A_{3}$. The results are as follows in Table 5.

\begin{tabular}{|c|l|l|l|}
\hline & \multicolumn{1}{|c|}{$\mathrm{A}_{1} \mathrm{P}$} & \multicolumn{1}{|c|}{$\mathrm{A}_{2} \mathrm{P}$} & \multicolumn{1}{|c|}{$\mathrm{A}_{3} \mathrm{P}$} \\
\hline $\mathrm{S}_{1}(\phi)$ & 0.80666 & 0.753483 & 0.770859 \\
\hline $\mathrm{S}_{2}(\phi)$ & 0.840736 & 0.788069 & 0.808633 \\
\hline $\mathrm{S}_{3}(\phi)$ & 0.766473 & 0.703639 & 0.743099 \\
\hline $\mathrm{S}_{4}(\phi)$ & 0.840736 & 0.788069 & 0.808633 \\
\hline $\mathrm{S}_{5}(\phi)$ & 0.761105 & 0.682104 & 0.712949 \\
\hline $\mathrm{S}_{6}(\phi)$ for $\mathrm{p}=2$ & 0.821222 & 0.776552 & 0.796418 \\
\hline $\mathrm{S}_{1}(\eta)$ & 0.916133 & 0.872675 & 0.885256 \\
\hline $\mathrm{S}_{2}(\eta)$ & 0.938333 & 0.9025 & 0.911667 \\
\hline $\mathrm{S}_{3}(\eta)$ & 0.89835 & 0.847525 & 0.865842 \\
\hline $\mathrm{S}_{4}(\eta)$ & 0.938333 & 0.9025 & 0.911667 \\
\hline $\mathrm{S}_{5}(\eta)$ & 0.9075 & 0.85375 & 0.8675 \\
\hline $\mathrm{S}_{6}(\eta)$ for $\mathrm{p}=2$ & 0.918319 & 0.877512 & 0.891129 \\
\hline
\end{tabular}

Table 5: Application to Medical diagnoses.

From the above table 5 it is clear that patient $\mathrm{P}$ can be diagnosed with viral fever.

\section{Conclusion}

Entropy, distance and similarity measure are significant research area in fuzzy information theory as they are efficient tools to deal with uncertain and insufficient information. Here we have derived new definition of entropy based on distance measure by considering degree of hesitancy in to account and derived relation between distance, entropy and similarity measures under IvIFE. Further, we have compared the derived similarity measures with some of the existing similarity measure and instances are used to show that the derived measures are able to draw conclusion when existing measures give the same result. Thereafter, proposed measures of similarity are applied to recognition of patterns and medical diagnoses.

\section{References}

[1] Atanassov, K.(1986), Intuitionistic fuzzy sets, Fuzzy Sets and Systems, 20 (1), 87-96. https://doi.org/10.1016/S0165-0114(86)80034-3

[2] Atanassov, K. T. (1999). Intuitionistic Fuzzy Sets. Heidelberg, New York: Physica-Verlag. https://doi.org/10.1007/978-3-7908-1870-3

[3] Atanassov, K. , \& Gargov, G.(1989) . Intervalvalued intuitionistic fuzzy sets, Fuzzy Sets and Systems, 31 (3) , 343-349. https://doi.org/10.1016/0165-0114(89)90205-4

[4] Dammak, F. Baccour, L and Alimi, A.M.(2016), An Exhaustive Study of Possibility Measures of Interval-valued Intuitionistic Fuzzy Sets abd Application to Multicriteria Decision Making, Advances in Fuzzy Systems, Vol 2016, Article ID 9185706, 10 pages. http://dx.doi.org/10.1155/2016/9185706

[5] De Luca A.,\& Termini S. (1972). A definition of a non-probabilistic entropy in setting of fuzzy sets. Information and Control, 20, 30. 
https://doi.org/10.1016/S0019-9958(72)9019941-312.

[6] Gau, W.L. ,\& Buehrer, D.J.(1993), Vague sets, IEEE Transactions on systems, Man and Cybernetics, 23 (2), 610-614. https://doi.org/10.1109/21.229476

[7] Grzegorzewski, P. (2004). Distances between intuitionistic fuzzy sets and/or interval-valued fuzzy sets based on the hausdorff metric. Fuzzy Sets and Systems, 48, 319-328. https://doi.org/10.1016/j.fss.2003.08.005

[8] Hu, K. \& Li, J. (2013), The entropy and similarity measure of interval valued intuitionistic fuzzy sets and their relationship, International Journal of Fuzzy Systems, Vol. 15(3), 279-288.

[9] Hung, W., \& Yang, M., (2006), Fuzzy entropy in intuitionistic fuzzy sets, International Journal of Intelligent Systems, Vol.21, 443-451. https://doi.org/10.1002/int.20131

[10] Kacprzyk, J. (1997). Multistage Fuzzy Control. Chichester: Wiley.

[11]Liu, X.C.(1992), Entropy, distance measure and similarity measure of fuzzy sets and their relations, Fuzzy Sets and Systems, 52, 305-318. https://doi.org/10.1016/0165-0114(92)90239-Z

[12] Park, J. H., Lin, K.M., Park, J.S. \& Kwun, Y.C. (2007), Distance between interval-valued intuitionistic fuzzy sets, Journal of Physics: Conference Series 96. https://doi.org/10.1088/1742-6596/96/1/012089

[13] Singh, P.(2012), A new method on measure of similarity between interval-valued intuitionistic fuzzy sets for pattern recognition, Journal of Applied \& Computational Mathematics, Vol.1(1), 1-5. https://doi.org/10.4172/2168-9679.1000101

[14] Sun, M. \& Liu, J.,(2012), New entropy and similarity measures for interval-valued intuitionistic fuzzy set, Journal of information \& Computational Sciences, vol.9(18), 5799-5806.

[15] Szmidt, E., \& Kacprzyk, J. (2000). Distance between intuitionistic fuzzy sets. Fuzzy Sets and Systems, 114(3), 505-518. https://doi.org/10.1016/S0165-0114(98)00244-9

[16] Tiwari, P. \& Gupta, P. (in press), Generalized Interval-valued Intuitionistic Fuzzy Entropy with some Similarity Measures, International Journal of Computing Science and Mathematics.

[17] Wei, C.P. , Wang, P. , \& Zhang, Y.Z. (2011), Entropy, similarity measures of interval-valued intuitionistic fuzzy sets and their applications, Inform. Sci., 181, 4273-4286. https://doi.org/10.1016/j.ins.2011.06.001

[18] Wu, C., Luo, P., Li, Y. \& Ren, X. (2014), A new similarity measure of interval-valued intuitionistic fuzzy sets considering its hesitancy degree and applications in expert systems, Mathematical Problems in Engineering. http://dx.doi.org/10.1155/2014/359214
[19] Xia, M. \&Xu, Z. (2010), Some new similarity measures for intuitionistic fuzzy values and their application in group decision making , Journal of Systems Science and System Engineering, Vol. 19(4), 430-452. https://doi.org/10.1007/s11518-010-5151-9

[20] Xu, Z. (2007a), On similarity measures of interval-valued intuitionistic fuzzy sets and their application to pattern recognitions, Journal of Southeast University (English Edition) 23 (1), 139-143.

[21] Xu, Z. (2007 b), Some similarity measures of intuitionistic fuzzy sets and their application to multiple attribute decision making, Fuzzy Optimization and Decision Making, Vol. 6(2), 109-121. https://doi.org/10.1007/s10700-007-9004-Z

[22] Xu, Z.S. \&Yager, R. R., (2009), Intuitionistic and interval valued intuitionistic fuzzy preference relations and their measures of similarity for the evaluation of agreement within a group, Fuzzy Optimization and Decision Making, Vol. 8(2), 123-139. https://doi.org/10.1007/s10700-009-9056-3

[23] Yang, Y., \& Chiclana, F. (2012), Consistency of 2D and 3D distances of intuitionistic fuzzy sets, Expert Systems with Applications, Vol. 39(10), 8665-8670. https://doi.org/10.1016/j.eswa.2012.01.199

[24] Yang, Y.J. \& Hinde, C. (2010), A new extension of fuzzy sets using rough sets: Rfuzzy sets, Information Sciences, 180, 354-365. https://doi.org/10.1016/j.ins.2009.10.004

[25] Ye, J. (2012), Multicriteria decision making method using the Dice similarity measure based on the reduct intuitionistic fuzzy sets of intervalvalued intuitionistic fuzzy sets, Applied Mathematical Modelling, vol.36(9), 4466-4472. https://doi.org/10.1016/j.apm.2011.11.075

[26]Zadeh, L. A.(1965), Fuzzy sets, Information and Control 8 (3), 338-356. https://doi.org/10.1016/S0019-9958(65)90241$\mathrm{X}$

[27]Zadeh, L.A.(1975), The concept of a linguistic variable and its application to approximate reasoning-I, Information Science, 8, 199-249. https://doi.org/10.1016/0020-0255(75)90036-5

[28]Zang, Q.S., Jiang, S.Y., Jia, B. G., \& Luo, S. H., (2010), Some information measures for intervalvalued intuitionistic fuzzy sets, Information Sciences, Vol.180, 5130-5145. https://doi.org/10.1016/j.ins.2010.08.038

[29]Zhang, Y.J., Ma, P.J., Su, H.,\& Zhang, C.P.(2011), Entropy on interval-valued intuitionistic fuzzy sets and its application in multi-attribute decision making, 2011 proceedings of 14th International Conference on Fusion (FUSION),1-7.

[30]Zhang, S. Li, X. \& Meng, F.(2016), An Approach to Multi-criteria Decision Making under Interval-valued Intuitionistic Fuzzy Vales 
and Interval Fuzzy Measures, Journal of Industrial and Production Engineering, Vol 33(4).

https://doi.org/10.1080/21681015.2016.1146362

[31]Zhang, Q., Xing, H., Liu, F., Ye, J. \& Tang, P. (2014), Some entropy measures for interval valued intuitionistic fuzzy sets based on distances and their relationships with similarity and inclusion measures, Information Sciences, 283, 55-69.

https://doi.org/10.1016/j.ins.2014.06.012

[32]Zhang, H., Zhang, W., \& Mei, C. (2009), Entropy of interval-valued fuzzy sets based on distance and its relationship with similarity measure, Knowledge-Based Systems, Vol. 22, 449-454.

https://doi.org/10.1016/j.knosys.2009.06.007 
